International Journal of Modern Physics D,

(C) World Scientific Publishing Company

\title{
THERMAL QUASI-EQUILIBRIUM STATES ACROSS LANDAU HORIZONS IN THE EFFECTIVE GRAVITY OF SUPERFLUIDS
}

\author{
Uwe R. Fischer \\ Eberhard-Karls-Universität Tübingen, Institut für Theoretische Physik \\ Auf der Morgenstelle 14, D-72076 Tübingen, Germany \\ Grigori E. Volovik* \\ L. D. Landau Institute for Theoretical Physics \\ Kosygin Str. 2, Russian Academy of Sciences, 117940 Moscow, Russia \\ Received (received date) \\ Revised (revised date)
}

\begin{abstract}
We give an account of the physical behaviour of a quasiparticle horizon due to nonLorentz invariant modifications of the effective space-time experienced by the quasiparticles ("matter") for high momenta. By introducing a "relativistic" conserved energymomentum tensor, we derive quasi-equilibrium states of the fluid across the "Landau" quasiparticle horizon at temperatures well above the quantum Hawking temperature. Nonlinear dispersion of the quasiparticle energy spectrum is instrumental for quasiparticle communication and exchange across the horizon. It is responsible for the establishment of the local thermal equilibrium across the horizon with the Tolman temperature being inhomogeneous behind the horizon. The inhomogeneity causes relaxation of the quasi-equilibrium states due to scattering of thermal quasiparticles, which finally leads to a shrinking black hole horizon. This process serves as the classical thermal counterpart of the quantum effect of Hawking radiation and will allow for an observation of the properties of the horizon at temperatures well above the Hawking temperature. We discuss the thermal entropy related to the horizon. We find that only the first nonlinear correction to the energy spectrum is important for the thermal properties of the horizon. They are fully determined by an energy of order $E_{\text {Planck }}\left(T / E_{\text {Planck }}\right)^{1 / 3}$, which is well below the Planck energy scale $E_{\text {Planck }}$, so that Planck scale physics is not involved in determining thermal quantities related to the horizon.
\end{abstract}

\section{Introduction}

The quantum phenomenon of Hawking radiationt is of kinematical origin, and relies on the existence of a Lorentzian signature background metric for the propagating particle, i.e., it depends upon the causal structyre of space-time. It does not depend on the field equations determining the metric日 and is, in particular, invariant under conformal transformations of the metricl. Lorentz invariance, which

*Present address: Helsinki University of Technology, Low Temperature Laboratory, P.O. Box 2200, FIN-02015 HUT, Finland. 
is a symmetry of nature experienced to hold on large enough scales, may not be an exact symmetry on the smallest scales. Because a particle at an infinite distance from the black hole horizon experiences an infinite blueshift when traced back to the horizon, the horizon is a suitable means to probe the high frequency structure of particle spectra: Black hole physics is necessarily being influenced by the large momentum modes of the quantum fields.

To trace the influence of the ultraviolet region upon the Hawking radiation from the black hole horizon, the condensed matter analogue of a black hole provides an orientation guide, with known underlying physical laws 2. In condensed matter, ultraviolet deviations from "Lorentz invariance" naturally arise, since the Lorentz symmetry itself is the consequence of the linear spectrum of excitations and thus exists only in the low energy corner. This allows for the use of physically imposed deviations from Lorentz invariance, which are governed by the Planck energy scale. In a number of papers, therefore, nonlinear dispersion of the quasiparticle spectrum has been employed to investigate the quantum physical behaviour related to the black hole horizon 5 . 6 , 8 . Below, we will find that in the most important cases only the first nonlinear correction determines the physics of the horizon, since the largest energies involved, although being determined by the Planck energy, are everywhere much smaller than the Planck scale.

Though initially the condensed matter analogue of black hole has been introduced in a normal liquid, it appeared that the properties of the quantum vacuum can be incorporated properly only if one uses quantum superfluid liquids 19,11 . The superfluid ground state moving without friction, and an inhomogeneous superfluid velocity $\mathbf{v}_{\mathbf{s}}$, provide the quantum vacuum, whose inhomogeneity serves as the gravitational field background. The matter propagating in the presence of this background is represented by fermionic (in Fermi superfluids) or bosonic (in Bose superfluids) quasiparticle excitations above the superfluid ground state. They form the normal component of the liquid. Two fluid hydrodynamics, introduced by Landau and Khalatnikov, incorporates the motion of the superfluid background and excitations 12 , and forms the counterpart of the Einstein equations, which incorporate both gravity and matter. A closer equivalence reveals itself when the quasiparticles possess "relativistic" properties in their low energy corner, so that the superfluid background provides an "acoustic" metrid of Lorentzian signature for the low energy excitations. This occurs for example in the case of Bogoliubov fermionic quasiparticles in ${ }^{3} \mathrm{He}-\mathrm{A}\left[\mathrm{B}\right.$, and phonons in superfluid ${ }^{4} \mathrm{He}$, as well as for excitations in dilute Bose condensates. The spectrum deviates from Lorentz invariance for larger energies in a well-defined way, fixed by the exact Bogoliubov spectrum in ${ }^{3} \mathrm{He}-\mathrm{A}$, respectively the phonon-maxon-roton spectrum in ${ }^{4} \mathrm{He}$. In ${ }^{3} \mathrm{He}-$ A, the analogy is even more attractive, since gravity arises naturally among the other low energy collective modes of the superfluid vacuum 11 , in the same manner as in Sakharov's theory of induced gravity 13 .

According to Landau's theory of superfluidity, the motion of the superfluid vacuum is frictionless until a critical velocity is reached, at which quasiparticle creation 
from the vacuum becomes possible. In superfluids with "relativistic"-like spectrum of quasiparticles, the Landau critical velocity equals the corresponding "speed of light" relevant for quasiparticles, which is the linear slope of the energy spectrum. For a generic profile of the superfluid velocity $\mathbf{v}_{\mathbf{s}}$-field, "superluminal" motion of the superfluid vacuum leads to formation of a horizon for "relativistic" quasiparticles. We will call such a horizon "Landau horizon". If the boundaries of the container are situated far enough away from the horizon, the quasiparticle creation predicted by Landau for superluminal flow occurs by a quantum process at the Landau horizon analogous to the Hawking radiation process at a gravity horizon.

The value of the Hawking temperature $T_{\mathrm{H}}=(\hbar / 2 \pi) \kappa_{\mathrm{S}}$ is determined by the surface acceleration $\kappa_{\mathrm{S}}$ on the black hole horizon. In the black hole analogy of fluids 2 . horizon normal, so that for purely radial flow $T_{\mathrm{H}}=\left.(\hbar / 2 \pi)\left(d v_{\mathrm{s}} / d r\right)\right|_{\text {hor }}$. In the presence of a Landau horizon in superfluids, Hawking radiation leads to quantum friction - the superfluid motion is decelerated due to dissipation caused by the Hawking radiation process 19,4 . 4 .

The value of the Hawking temperature serves also as a crossover measure to distinguish the region of conventional thermodynamics of the quasiparticle gas at $T \gg T_{\mathrm{H}}$ and the quantum regime $T<T_{\mathrm{H}}$, for which dimensional quantization of the quasiparticle energy levels becomes important. The Hawking temperature is typically rather small even for condensed matter analogues of the horizon, which makes it difficult to observe quantum effects. Therefore, it will prove useful to discuss those thermal consequences of the horizon at $T \gg T_{\mathrm{H}}$ which can be simulated by the thermal states of quasiparticles in the presence of a flowing superfluid background.

In the absence of a Landau horizon the true equilibrium thermal states (i.e. those with constant temperature and without entropy production) exist for any velocity field $\mathbf{v}_{\mathrm{s}}$. They correspond to Tolman's law in a gravity field with constant Tolman temperature, cf.15, $\S 129$. In the presence of the Landau horizon the situation changes: True equilibrium states no longer exist. This means that any thermal state of superfluid liquid is dissipative in the presence of a horizon. We find such dissipative quasi-equilibrium thermal states across the Landau horizon using the simplest example of superflow in $1+1$ space-time dimensions. They are characterized by constant true temperature $T$ outside the horizon (Tolman's law in gravity), and varying $T$ behind it, which satisfies a modified Tolman law. The physics of these states is determined by deviations from Lorentz invariance in the ultraviolet region, in which quasiparticle excitations can propagate superluminally. The superluminal propagation across the horizon allows quasiparticles to exchange information and energy, and thus ensures the continuity of the temperature across the horizon. At $T \gg T_{\mathrm{H}}$, the dissipation caused by quantum effects related to Hawking radiation can be neglected, and the relaxation (and final extinction) of the black hole occurs via entropy production behind the horizon, where the "Tolman temperature" is not constant. The dissipation is caused by the thermal conductivity and viscosity of 
the excitation gas (the normal component of the liquid).

The high energy corrections to the quasiparticle spectrum are responsible for the processes of dissipation in our $1+1 \mathrm{~d}$ case. These processes are absent in the purely relativistic régime of massless excitations, if the so-called Conformal Killing Vector

$(\mathrm{CKV})$ conditions are satisfied, see, e.g. 16. These CKV conditions are realized for the $1+1 \mathrm{~d}$ case, where thus dissipation can be caused only by deviations from Lorentz invariance in the ultraviolet region. Dissipation is most pronounced in the vicinity of the horizon due to the blueshift for quasiparticles there, so that the horizon region is responsible for the largest part of the entropy production. This dissipation process represents the classical counterpart of the quantum process of entropy production by Hawking radiation from the horizon. The same high energy corrections relevant for entropy production determine also the entropy of the horizon itself.

In the second section to follow, we review the properties of the fermionic quasiparticle spectrum in ${ }^{3} \mathrm{He}-\mathrm{A}$ and the bosonic one in ${ }^{4} \mathrm{He}$. This comprises the influence on the energy spectrum of the superfluid background as an effective gravity field, the formation of the Landau horizon and the structure of the superfluid vacuum in the presence of a horizon, taking into account the nonlinear dispersion at higher energy and, finally, the Hawking radiation process in the presence of such a dispersion.

In the third section, using the "acoustic" Painlevé-Gullstrand metric, we reformulate the hydrodynamic equations for the normal component in a "relativistically" invariant form, so that they correspond to the thermodynamics of massless 1+1dimensional relativistic particles moving in a gravitational field.

The thermal states across the horizon are obtained as solutions of the hydrodynamic equations on both sides of the horizon in the fourth section, with matching between the states occuring due to non-Lorentzian corrections, which are most pronounced near the Landau horizon. While the equilibrium thermal state outside the horizon is determined as usual by a timelike Killing vector, the thermal state behind the horizon satisfies the general equilibrium condition generated by a spacelike conformal Killing vector.

In section five the entropy related to the thermal states close to the horizon is calculated, and dissipation behind and at the horizon is discussed. To compare the thermal and quantum processes of the black hole relaxation, we consider the quantum correction to the thermal energy in our flow geometry. It has a negative sign and thus, as usually assumed, can compensate the diverging term in the stressenergy tensor for the Hartle-Hawking state at $T=T_{\mathrm{H}}$. However, we argue that this state nevertheless remains dissipative due to the nonlinear dispersion at high energy, so that the black hole horizon must eventually shrink at any temperature.

\section{Spectrum and motion of quasiparticles}

\subsection{Phonons in a moving superfluid}

A suitably simple framework for our purposes is a superfluid with massless exci- 
tations, which have a relativistic-like spectrum at low energy. There are two classes of such systems: bosonic and fermionic superfluids. A bosonic superfluid is a degenerate Bose system which experiences Bose-Einstein condensation. In the low energy limit there are only bosonic excitations in such liquids. In the very low energy corner these are phonons, whose energy spectrum is $E^{\mathrm{com}}=c p$, where $c$ is the speed of sound, and energy is measured in the reference frame comoving with the superfluid condensate. This is realized in superfluid ${ }^{4} \mathrm{He}$ and in laser-manipulated, ultracold Bose-Einstein condensed gases. At higher momentum $p$ the energy spectrum deviates from the relativistic linear behaviour:

$$
E^{\mathrm{com}}=c p+\gamma p^{3}+\ldots \equiv c p\left(1 \pm \frac{1}{2}\left(\frac{c p}{E_{\text {Planck }}}\right)^{2}+\ldots\right)
$$

where $\gamma$ can be positive or negative. The parameter $\gamma$ is typically determined by the analog of the Planck energy $E_{\text {Planck }}$ in superfluids. It will be shown, for positive $\gamma$, that while the first nonlinear correction is important for the horizon problem, the higher order corrections are in many cases small and can be neglected. In these cases "transPlanckian" physics is not involved, i.e., no "quantum gravity" is necessary.

The excitation energy in the laboratory frame, in which the superfluid velocity $\mathbf{v}_{\mathbf{s}}$, the momentum and the energy of quasiparticles are measured, is obtained by a Galilean transformation which has the form

$$
\mathbf{p}=\mathbf{p}^{\mathrm{lab}}=\mathbf{p}^{\mathrm{com}}, \quad E=E^{\mathrm{lab}}(\mathbf{p})=E^{\mathrm{com}}(\mathbf{p})+\mathbf{p} \cdot \mathbf{v}_{\mathrm{s}},
$$

or, using (1),

$$
\left(E-\mathbf{p} \cdot \mathbf{v}_{\mathrm{s}}\right)^{2}=c^{2} p^{2}+2 \gamma c p^{4}+\ldots \equiv c^{2} p^{2}\left(1 \pm\left(\frac{c p}{E_{\text {Planck }}}\right)^{2}\right)+\ldots
$$

This equation for the spectrum corresponds to what we would obtain for a plane wave mode from a relativistic scalar wave equation in the WKB approximation, modified in the comoving frame by higher order derivatives to include nonlinear dispersionl. If we neglect nonlinear corrections, we can write the energy dispersion (3) in the form $g^{\mu \nu} p_{\mu} p_{\nu}=0$, where the contravariant components of the metric are $g^{00}=-1, g^{0 i}=-v_{\mathrm{s}}^{i}, g^{i j}=c^{2} \delta^{i j}-v_{\mathrm{s}}^{i} v_{\mathrm{s}}^{j}$. In this Lorentz invariant limit, $c p \ll E_{\text {Planck }}$, the phonons become "relativistic" massless particles of energy $E=$ $-p_{0}$, propagating in a $(3+1)$-dimensional space-time background with metric $g_{\mu \nu}$ of Lorentzian signature. This effective metric takes the Painlevé-Gullstrand form

$$
d s^{2}=-\left(1-\left(v_{\mathrm{s}} / c\right)^{2}\right) d t^{2}-2 c^{-2} \mathbf{v}_{\mathrm{s}} \cdot d \mathbf{r} d t+c^{-2}(d \mathbf{r})^{2},
$$

with $\sqrt{-g}=c^{-3}$. Observe that we define the metric coefficients such that the space-time interval has dimension of time, so that the quasiparticle energy has the same dimension as $p_{0}$. 


\subsection{Landau critical velocity, ergosurface and Landau horizon}

If the superfluid velocity exceeds the Landau critical value

$$
v_{L}=\min \frac{E^{\mathrm{com}}(\mathbf{p})}{p}
$$

the energy $E=E^{\text {lab }}(\mathbf{p})$ of some excitations, as measured in the laboratory frame, becomes negative. This allows for excitations to be nucleated from the vacuum. For a superfluid velocity field which is stationary in the laboratory frame, the surface $v_{\mathrm{s}}(\mathbf{r})=v_{L}$, which bounds the region where quasiparticles can have negative energy, the ergoregion, is called the ergosurface.

If the dispersion bends upwards, $\gamma>0$, the Landau critical velocity coincides with the "speed of light", $v_{L}=c$, so that the ergosurface is determined by $v_{\mathrm{s}}(\mathbf{r})=$ c. In the Lorentz invariant limit, this corresponds to $g_{00}(\mathbf{r})=0$, which is just the definition of the ergoregion in gravity. In the case of radial flow (zero axial component of $\mathbf{v}_{\mathbf{s}}$ ) of the superfluid vacuum towards the origin, the ergosurface becomes the horizon in the Lorentz invariant limit, and the region inside the horizon simulates a black hole for low energy phonons. Strictly speaking this is not a true horizon for phonons: Due to the nonlinear dispersion, their group velocity $v_{g}=d E^{\mathrm{com}} / d p=c+3 \gamma p^{2}>c$, and thus the high energy quasiparticles are allowed to leave the black hole region. It is, hence, a horizon only for quasiparticles living exclusively in the very low energy corner, which are not aware of the possibility of "superluminal" motion.

In the case of negative dispersion, $\gamma<0$, the group velocity $v_{g}=d E^{\mathrm{com}} / d p<c$. In such superfluids the relativistic ergosurface $v_{\mathrm{s}}(\mathbf{r})=c$ does not coincide with the true ergosurface, which is determined by $v_{\mathrm{s}}(\mathbf{r})=v_{L}<c$. In superfluid ${ }^{4} \mathrm{He}$, where $\gamma<0$, the Landau velocity is related to the roton part of the spectrum, and is about four times less than $c .^{\dagger}$ For inward radial flow, the ergosphere occurs at $v_{\mathrm{S}}(r)=v_{L}<c$, while the inner surface $v_{\mathrm{S}}(r)=c$ still marks the horizon, in contrast to relativistically invariant systems, for which ergosurface and horizon coincide for purely radial flow.

\subsection{Painlevé-Gullstrand versus Schwarzschild metric in effective gravity}

In our context, it appears worthwhile to point out that in the effective theory of gravity, which occurs in condensed matter systems in the form that the primary quantity is the contravariant metric tensor $g^{\mu \nu}$ describing the energy spectrum, two seemingly equivalent representations of the black hole metric, in terms of either the Schwarzschild or the Painlevé-Gullstrand line elements, are in fact not equivalent in terms of the required stability of the underlying superfluid vacuum. Let us construct an isotropic Galilean superfluid in $3+1$ dimensions, in such a way that

${ }^{\dagger}$ The phonon dispersion in superfluid ${ }^{4} \mathrm{He}$ actually first has a slight upturn of phonon dispersion (and hence $\gamma>0$ ) for small phonon momenta. If we assign $\gamma<0$, it is thus meant that the dispersion after this slight upturn, for intermediate momenta, bends down to the roton minimum. 
the low energy spectrum of the Bogoliubov quasiparticles in the frame comoving with the superfluid is given by

$$
\left(E^{\mathrm{com}}\right)^{2}=c^{2} p^{2},
$$

where $c$ is the parameter which plays the part of the speed of light. Then, for spherically symmetric velocity profiles $v_{\mathrm{s}}(r)=\mp c \sqrt{r_{\mathrm{S}} / r}$, the Painlevé-Gullstrand line elements are obtained in the laboratory frame as:

$$
\begin{aligned}
& d s^{2}=-\left(1-\frac{r_{\mathrm{S}}}{r}\right) d t^{2}+2 c^{-1} \sqrt{\frac{r_{\mathrm{S}}}{r}} d r d t+c^{-2} d r^{2}+\left(r^{2} / c^{2}\right) d \Omega^{2}, \\
& d s^{2}=-\left(1-\frac{r_{\mathrm{S}}}{r}\right) d t^{2}-2 c^{-1} \sqrt{\frac{r_{\mathrm{S}}}{r}} d r d t+c^{-2} d r^{2}+\left(r^{2} / c^{2}\right) d \Omega^{2}
\end{aligned}
$$

where $r_{\mathrm{S}}$ is the Schwarzschild radius. The Painlevé-Gullstrand line elements in (7,8) describe black hole and white hole analogues (see e.g. ref.2, a pedagogical review of the Painlevé-Gullstrand metric is contained in 17 ). The "surface gravity" at the Schwarzschild radius is, therein, $\kappa_{\mathrm{s}}=d v_{\mathrm{s}} /\left.d r\right|_{r_{\mathrm{S}}}=c / 2 r_{\mathrm{S}}$, and the Hawking temperature which follows $T_{\mathrm{H}}=\hbar \kappa_{\mathrm{s}} / 2 \pi$.

An "equivalent" representation of the black or white hole metric is given by the Schwarzschild line element, which in terms of the same superfluid velocity reads

$$
d s^{2}=-\left(1-v_{\mathrm{s}}^{2} / c^{2}\right) d \tilde{t}^{2}+\frac{d r^{2}}{c^{2}-v_{\mathrm{s}}^{2}}+\left(r^{2} / c^{2}\right) d \Omega^{2} .
$$

They are related by the following coordinate transformation:

$$
\tilde{t}(r, t)=t+\left(\frac{2}{v_{\mathrm{s}}(r)}+\ln \frac{1-v_{\mathrm{s}}(r)}{1+v_{\mathrm{s}}(r)}\right), \quad d \tilde{t}=d t+\frac{v_{\mathrm{s}}}{1-v_{\mathrm{s}}^{2}} d r .
$$

From the point of view of the effective gravity in superfluids, (7) and (8) describe the dynamics of quasiparticles propagating in the superfluid vacuum, moving with superfluid velocity $\mp\left|v_{\mathrm{s}}(r)\right|$, directed to the origin and away from the origin, respectively. The space-time $(t, \mathbf{r})$ is the absolute space-time of the laboratory frame, i.e., as measured by the external experimentalist living in the real world of the laboratory. The squared energy spectrum of the quasiparticles, determined by the contravariant components of the metric (77,8), is obtained to be

$$
\left(E-v_{\mathrm{s}}(r) p_{r}\right)^{2}=c^{2} p^{2}
$$

or, solving for $E$,

$$
E=v_{\mathrm{s}}(r) p_{r} \pm c p
$$

The time $\tilde{t}$ is the time as measured by the "inner" observer at "infinity" (far from the hole). The "inner" means that this observer "lives" in the superfluid background and makes use of "relativistic" massless quasiparticles (phonons or other excitations) to synchronize clocks. The inner observer at some point $R \gg 1$ sends a quasiparticle 
pulse at the moment $t_{1}$ which arrives at point $r$ of $t=t_{1}+\int_{r}^{R} d r^{\prime} /\left|v_{-}\right|$absolute (laboratory) time, where $v_{+}$and $v_{-}$are absolute (laboratory) velocities of radially propagating quasiparticles, moving outwards and inwards respectively:

$$
v_{ \pm}=\frac{d r}{d t}=\frac{d E}{d p_{r}}= \pm 1+v_{\mathrm{s}}(r) .
$$

Since from the point of view of the inner observer the speed of light (the propagation speed of quasiparticles) is an invariant quantity, and does not depend on the direction of propagation, for him or her the moment of arrival of the pulse to $r$ is not $t$ but $\tilde{t}=\left(t_{1}+t_{2}\right) / 2$, where $t_{2}$ is the time when the pulse reflected from $r$ returns to the observer at $R$. Since $t_{2}-t_{1}=\int_{r}^{R} d r^{\prime} /\left|v_{-}\right|+\int_{r}^{R} d r^{\prime} /\left|v_{+}\right|$, one obtains for the time measured by an inner observer

$$
\begin{aligned}
\tilde{t}(r, t) & =\frac{t_{1}+t_{2}}{2}=t+\frac{1}{2}\left(\int_{r}^{R} \frac{d r^{\prime}}{v_{+}}+\int_{r}^{R} \frac{d r^{\prime}}{v_{-}}\right) \\
& =t+\left(\frac{2}{v_{\mathrm{s}}(r)}+\ln \frac{1-v_{\mathrm{s}}(r)}{1+v_{\mathrm{s}}(r)}\right)-\left(\frac{2}{v_{\mathrm{s}}(R)}+\ln \frac{1-v_{\mathrm{s}}(R)}{1+v_{\mathrm{s}}(R)}\right),
\end{aligned}
$$

which is just (10), up to a constant shift.

At $r=r_{\mathrm{S}}=1$ one has an event horizon, where the superfluid velocity crosses the "speed of light" $c \equiv 1$. If the fluid moves towards the origin, that is $v_{\mathbf{s}}(r)<0$, this velocity field reproduces the horizon of the black hole (the so-called sonic black holet ): Since the velocity of the fluid behind horizon exceeds the speed $c$ of the propagation of quasiparticles with respect to the fluid, the low energy quasiparticles are trapped within the horizon. If the fluid moves from the origin outwards, $v_{\mathrm{s}}(r)>$ 0 , the velocity field reproduces a white hole horizon.

In the (necessarily) complete physical space-time of the laboratory, an external observer can detect quasiparticles radially propagating into (but not out of) the black hole, or out of (but not into) the white hole. The energy spectrum of the quasiparticles remains to be well determined both outside and inside the horizon. Quasiparticles cross the black hole horizon with the absolute velocity $v_{-}=-1-v_{\mathrm{s}}=$ -2 , i.e. with twice the speed of light: $r(t)=1-2\left(t-t_{0}\right)$. In the case of a white hole horizon one has $r(t)=1+2\left(t-t_{0}\right)$. In contrast, from the point of view of the inner observer the horizon cannot be reached and crossed: the horizon can be approached only asymptotically in infinite time: $r(\tilde{t})=1+\left(r_{0}-1\right) \exp (-\tilde{t})$. Such a disability of the local observer, who lives in the curved world of superfluid vacuum, happens because he or she is limited in his or her observations by the "speed of light", so that the coordinate frame he or she uses is seriously limited in the presence of a horizon and becomes incomplete.

The Schwarzschild metric (9) naturally arises for the inner observer, if the Painlevé-Gullstrand metric is an effective metric for quasiparticles in superfluids, but not vice versa. It can in principle arise as an effective metric; however, in the presence of a horizon such a metric indicates an instability of the underlying 
medium. To obtain a line element of the Schwarzschild metric as an effective metric for quasiparticles, the quasiparticle energy dispersion in the laboratory frame has to be

$$
E^{2}=c^{2}\left(1-\frac{r_{\mathrm{S}}}{r}\right)^{2} p_{r}^{2}+c^{2}\left(1-\frac{r_{\mathrm{S}}}{r}\right) p_{\perp}^{2} .
$$

In the presence of a horizon such a spectrum has sections of the transverse momentum $p_{\perp}$ having $E^{2}<0$. The consequence of an imaginary frequency of excitations signals the instability of the superfluid vacuum, if this vacuum exhibits the Schwarzschild metric as an effective metric for excitations: Quasiparticle perturbations may grow exponentially without bound in laboratory (Killing) time, like $\exp [t|\Im(E)|]$, destroying the superfluid vacuum. Nothing of this kind happens in the case of the Painlevé-Gullstrand line element, for which the quasiparticle energy is real even behind the horizon. The main difference between Painlevé-Gullstrand and Schwarzschild metrics as effective metrics is thus as follows. The first metric leads to the slow process of quasiparticle radiation from the vacuum at the horizon (Hawking radiation), whereas the second one indicates a crucial instability of the vacuum behind the horizon.

In general relativity, it is usually assumed that the two metrics can be converted into each other by the coordinate transformation in (10). In condensed matter, the coordinate transformation leading from one metric to another is not that innocent if an event horizon is present. The reason why the physical behaviour implied by the choice of metric representation changes drastically, is that the transformation between the two line elements, $t \rightarrow t+\int^{r} d r^{\prime} v /\left(c^{2}-v^{2}\right)$, is singular on the horizon, and thus can be applied only to a part of space-time. In condensed matter, only those effective metrics are physical which are determined everywhere in the real physical space-time and come from a physically reasonable energy spectrum of quasiparticles. The two representations of the "same" metric cannot, then, be strictly equivalent metrics, and we have different classes of equivalence, which cannot be transformed to each other by coordinate transformations which are regular everywhere. Painlevé-Gullstrand metrics for black and white holes are determined everywhere, but belong to two different classes. The transition between these two metrics occurs via the singular transformation $t \rightarrow t+2 \int^{r} d r^{\prime} v /\left(c^{2}-v^{2}\right)$, or via the Schwarzschild line element, which is prohibited in condensed matter physics, as explained above, since it is pathological in the presence of a horizon. It is not determined in the whole space-time, and is singular at the horizon.

Of importance is the fact that in the effective theory, there is no need for an additional extension of the space-time to make it geodesically complete. This is because the effective space-time is always incomplete (open), since it exists only in the low energy "relativistic" corner, and quasiparticles escape this space-time to a nonrelativistic domain when their energy increases beyond the relativistic linear approximation régime 10 .

\subsection{Bogoliubov fermions in Fermi superfluids}


Another class of quasirelativistic systems is provided by Fermi superfluids with point nodes in the spectrum, the physically realised example in an earth laboratory being the superfluid ${ }^{3} \mathrm{He}-\mathrm{A}$, and possibly chiral $p$-wave superconductors 19 . The fermionic quasiparticles in ${ }^{3} \mathrm{He}-\mathrm{A}$ are described by a Nambu space Hamiltonian which, in the locally comoving frame, has the form

$$
\hat{H}_{A}=\tau_{3} \epsilon(\mathbf{p})+\frac{\Delta_{A}}{p_{F}}\left[\tau_{1} p_{x}+\tau_{2} p_{y}\right], \quad \epsilon(\mathbf{p}) \approx \frac{p^{2}-p_{F}^{2}}{2 m^{*}} \approx v_{F}\left(p-p_{F}\right) .
$$

Here, $\Delta_{A}$ represents the amplitude of the gap, $p_{F}$ and $v_{F}=p_{F} / m^{*}$ are Fermi momentum and Fermi velocity respectively. We assume that the orbital momentum vector $\hat{\mathbf{l}}$ is oriented along the $z$ axis and that the absolute value of the momentum $p=|\mathbf{p}|$ is restricted by $p-p_{F} \ll p_{F}$.

The Bogoliubov spectrum of fermions in the comoving frame is obtained by squaring (16)

$$
\left(E^{\mathrm{com}}\right)^{2}=\hat{H}_{A}^{2}=\left(p^{2} / 2 m^{*}-p_{F}^{2} / 2 m^{*}\right)^{2}+c_{\perp}^{2}\left(p_{x}^{2}+p_{y}^{2}\right) .
$$

Here $c_{\perp} \equiv \Delta_{A} / p_{F} \ll v_{F}$ (the slope of the gap at the node), plays the role of the "velocity of light" in the direction perpendicular to $\hat{1}$.

If the superfluid velocity is restricted to lie in the plane perpendicular to $\hat{\mathbf{l}}$ and does not depend on $z$, the momentum projection $p_{z}$ is a conserved quantity, and one obtains a $2+1 \mathrm{~d}$ system with the laboratory frame energies

$$
\left(E-\mathbf{p}_{\perp} \cdot \mathbf{v}_{\mathrm{s}}\right)^{2}=\frac{\left(p_{\perp}^{2}-\tilde{p}_{F}^{2}\right)^{2}}{4 m_{*}^{2}}+c_{\perp}^{2} p_{\perp}^{2}, \quad \tilde{p}_{F}^{2} \equiv p_{F}^{2}-p_{z}^{2} .
$$

In the very low energy régime, i.e., if $\mathbf{p}$ is chosen to be close to $p_{F} \hat{\mathbf{e}}_{z}$, and in addition $p_{\perp}^{2}=p_{x}^{2}+p_{y}^{2} \ll m_{*}^{2} c_{\perp}^{2}$, we can write the energy dispersion (18) in a Lorentz invariant form with $(2+1)$-dimensional metric (neglecting the quasiparticle motion in the $z$ direction). This metric again takes the Painlevé-Gullstrand form

$$
d s^{2}=-\left(1-\left(v_{\mathrm{s}} / c_{\perp}\right)^{2}\right) d t^{2}-2 c_{\perp}^{-2} \mathbf{v}_{\mathbf{s}} \cdot d \mathbf{r} d t+c_{\perp}^{-2} d r^{2},
$$

with $\sqrt{-g}=c_{\perp}^{-2}$.

The Bogoliubov spectrum, say, for $p_{z}=p_{F}, \tilde{p}_{F}=0$ reads

$$
E=\mathbf{v}_{\mathrm{s}} \cdot \mathbf{p}_{\perp} \pm \sqrt{\left(\frac{p_{\perp}^{2}}{2 m_{*}}\right)^{2}+c_{\perp}^{2} p_{\perp}^{2}} .
$$

Squared, this has the same form as for phonons with positive dispersion in (3), with the "Planck" energy $E_{\text {Planck }}=2 m_{*} c_{\perp}^{2}$. This shows that the form of spectrum with cubic dispersion is generic for any system which has "relativistic" quasiparticles in the low energy corner. Further on, we shall consider the positive dispersion, $\gamma>0$.

\subsection{Physical vacua across the horizon}


Close to the horizon the horizon surface can be considered to be flat, so that the radial flow becomes a one-dimensional flow of superfluid along the normal to the horizon (along the $x$ coordinate direction), this flow depending only on $x\left(\mathbf{v}_{\mathrm{s}}=\right.$ $v_{\mathrm{s}}(x) \hat{\mathbf{x}}$, with $\left.v_{\mathrm{s}}<0\right)$. Since there is no dependence on $y$ and $z$, the momentum projections $p_{z}$ and $p_{y}$ of quasiparticles are fixed and one obtains $(1+1)$-dimensional motion of quasiparticles whose "mass" and "speed of light" are determined by $p_{z}$ and $p_{y}$. Figures 13 is display the energy spectrum for the case of zero "mass", which means $p_{y}=p_{z}=0$ for the Bose condensate, and $p_{y}=0, p_{z}= \pm p_{F}$ for ${ }^{3} \mathrm{He}-\mathrm{A}$. We measure $v_{\mathrm{s}}$ in units of $c_{\perp} ; E$ and $p_{x}$ in units of $m_{*} c_{\perp}^{2}=\Delta_{A}\left(c_{\perp} / v_{F}\right)$ and $m_{*} c_{\perp}=$ $\Delta_{A} / v_{F}$, which represent the crossover "Planck energy" and the crossover "Planck momentum" to the "nonrelativistic" domain, respectively. Normalised quantities in what follows will be designated by a tilde, save for $k \equiv p_{x} / m_{*} c_{\perp}$ and any velocities, which are understood to be scaled by $c_{\perp}$ (or $c$ in the case of the Bose condensate), in the normalised relation:

$$
\tilde{E}=v_{\mathrm{s}} k \pm \sqrt{k^{2}+\frac{k^{4}}{4}} .
$$

The position of the horizon is determined by $v_{\mathrm{s}}\left(x_{h}\right)=-1$. We remind the reader that, if the nonlinear dispersion of quasiparticles is positive, this is not a true horizon, since the group velocity in the comoving frame exceeds the "speed of light"

$$
\left|v_{g}^{\mathrm{com}}\right|=\frac{1+\frac{k^{2}}{2}}{\sqrt{1+\frac{k^{2}}{4}}}>1,
$$

and consequently high energy quasiparticles can propagate across the horizon.

The horizon is understood to be a "Landau horizon" - the position in space where the Landau criterium is first violated, i.e., where the superfluid velocity reaches the minimum of the comoving frame group velocity:

$$
v_{\mathrm{s}}\left(x_{h}\right)=-\min \left|v_{g}^{\mathrm{com}}\right|=-1 .
$$

Let us first discuss the vacuum for the Fermi system, which corresponds to filling of the negative energy states. The vacuum as viewed in the comoving frame corresponds to the filling of states with negative root in (20) (thick lines in Fig.11). Outside the horizon, where $\left|v_{\mathrm{s}}\right|<1$ (Fig.2), the vacuum in the laboratory frame coincides with the vacuum in the comoving frame: In both systems the negative energy levels are occupied. This, however, does not happen inside the horizon, where $\left|v_{\mathrm{s}}\right|>1$ (Fig.3), and some states, which have negative energy in the comoving frame, have positive energy in the laboratory frame. In gravity, the state corresponding to the vacuum in the comoving frame is the state empty of particles as seen by the "free fall" observer (who moves with superfluid velocity $v_{\mathrm{s}}$ ). The vacuum in the laboratory frame corresponds to the Boulware vacuum in gravity: It is empty for the observer at infinity. In the purely relativistic régime in $1+1$ dimension the vacuum behind the horizon, determined in the comoving frame, is obtained if the 


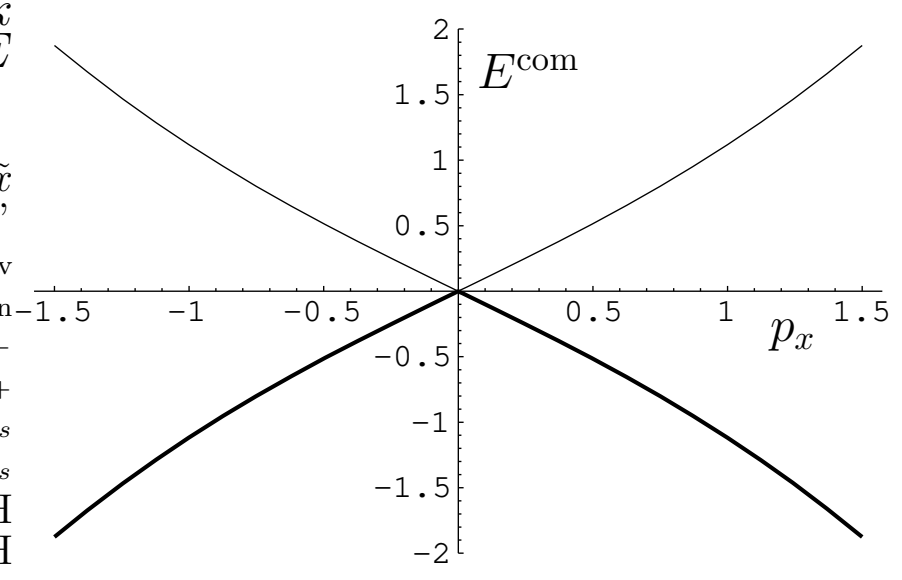

Figure 1: Energy spectrum in comoving frame. The occupied negative energy levels designated by thick lines form the comoving frame vacuum.

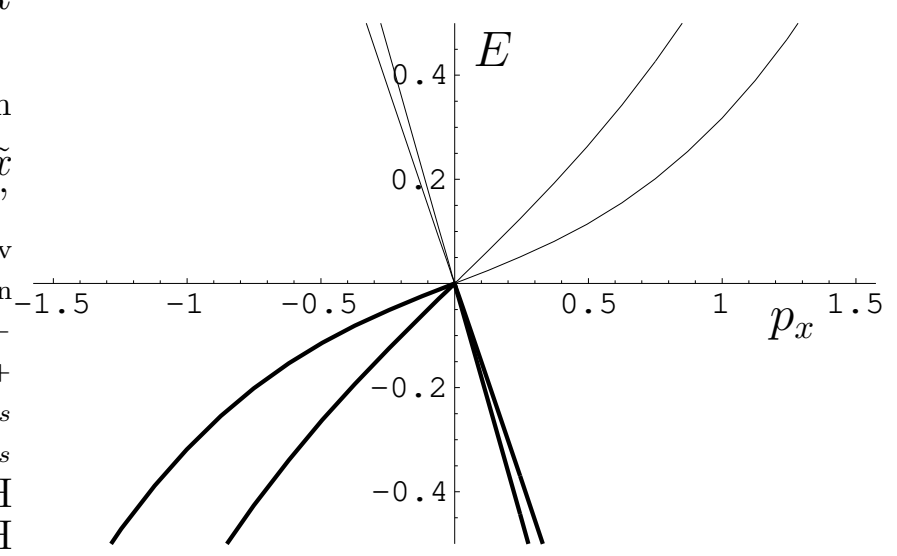

Figure 2: Spectrum in the laboratory frame outside the "horizon" at two points in space where $v_{\mathrm{s}}=-0.5$ and $v_{\mathrm{s}}=-0.8$.

(negative) momentum of upper index, $p^{1}=\left(1-v_{\mathrm{s}}^{2}\right) p_{1}-v_{\mathrm{s}} p_{0}=\left(1-v_{\mathrm{s}}^{2}\right) p+v_{\mathrm{s}} E$, is used as the Hamiltonian, $H=-p^{1}$, corresponding to the interchange of timelike and spacelike coordinates behind a horizon. The states with positive $p^{1}$ are occupied in this vacuum, and $p^{1}=-i\left[\left(1-v_{\mathrm{s}}^{2}\right) \partial_{x}-v_{\mathrm{s}} \partial_{t}\right]$ is a conformal Killing vector behind the horizon. The same Hamiltonian $H=-p^{1}$ determines thermal equilibrium (section 4.2.). However, any deviation from the linear relativistic régime leads to dissipation in this vacuum or the thermal equilibrium state.

Close to the horizon, the velocity profile can be linearized: $v_{\mathrm{s}}(x)=-1+$ 


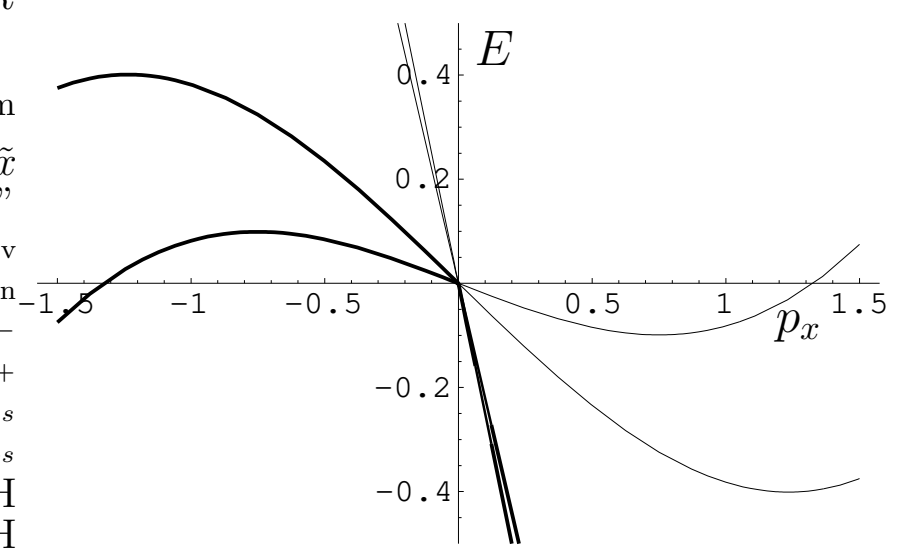

Figure 3: Spectrum in the laboratory frame inside the "Landau horizon" at two points where $v_{\mathrm{s}}=-1.2$ and $v_{\mathrm{s}}=-1.5$.

$\left(\kappa_{\mathrm{S}} / c_{\perp}\right) \delta x \equiv-1+\tilde{x}$, with $\delta x=x-x_{h} \ll c_{\perp} / \kappa_{\mathrm{s}}$. Since in the above considerations we assumed that $\tilde{E} \ll 1$ and $\tilde{x} \ll 1$, only small $k$ are relevant and we can approximately write

$$
k^{3} / 8+k \tilde{x} \simeq \tilde{E} .
$$

The same cubic dispersion has been discussed in 6 . From equation (24) it follows that the characteristic scales for $k, \tilde{E}^{\text {com }}$ and $\tilde{x}$ are $\tilde{E}^{1 / 3}, \tilde{E}^{1 / 3}$ and $\tilde{E}^{2 / 3}$, respectively. In dimensionful units these are

$$
\begin{array}{r}
E^{\mathrm{com}}=c p \sim E_{\text {Planck }}^{2 / 3} E^{1 / 3} \ll E_{\text {Planck }}, \\
\left|x-x_{h}\right| \sim \frac{c}{\kappa_{\mathrm{s}}}\left(\frac{E}{E_{\text {Planck }}}\right)^{2 / 3} \sim l_{\text {Planck }}\left(\frac{E}{T_{\mathrm{H}}}\right)^{2 / 3}\left(\frac{E_{\text {Planck }}}{T_{\mathrm{H}}}\right)^{1 / 3} \gg l_{\text {Planck }} .
\end{array}
$$

The characteristic energy, although highly blue-shifted compared with the laboratory energy $E$, is nevertheless much smaller than the Planck energy scale. Correspondingly, the characteristic region of the shell (membrane) in the vicinity of the horizon, though very narrow, is nevertheless large compared to the Planck length scale (the quasiparticle energy in the laboratory frame is determined either by the Hawking temperature, or by the temperature of the heat bath, in both cases $\left.E \geq T_{\mathrm{H}}\right)$. Consequently, the higher order corrections to the nonlinear energy spectrum are negligibly small, so that "transPlanckian" physics is not involved save for its providing the parameter $\gamma$, which can be considered as a phenomenological parameter in the effective low energy theory.

\subsection{Wave function}

If the initial state of the superfluid is the vacuum in the comoving frame, then in the presence of a horizon this state represents a false vacuum: It possesses large 
positive energy in the laboratory frame, so that it has to decay. If there is an interaction with the walls of the container, the relaxation occurs due to fast processes of the dynamics of the superfluid order parameter. However, if the interaction with walls is screened, or the wall is very far, the comoving inner observer loses the reference frame imposed by the walls. Now the velocity field itself is what the inner observer can observe. If the flow is homogeneous, there is no evidence for him or her that the superfluid is moving. Thus the only information comes from the inhomogeneity of the velocity field, which establishes a special frame, in which the velocity profile is time independent. This means that if a horizon is present, its relaxation is determined by the gradient of the superfluid velocity. One example is quantum Hawking radiation, with the Hawking temperature determined by the gradient of the velocity at the horizon. Another example is the classical thermal state in the presence of an horizon, whose relaxation is provided by the hydrodynamic entropy production induced by the velocity gradient.

Let us first recall the quantum relaxation. Using the energy $\tilde{E}(k)$ of (24) as a Hamiltonian, and canonically quantizing,

$$
[\tilde{x}, k]=i \tilde{\hbar}, \quad \tilde{\hbar}=\hbar \kappa_{\mathrm{s}} /\left(m_{*} c_{\perp}^{2}\right)
$$

we write the stationary Schrödinger equation in the momentum representation as $\left(\tilde{x}=i \tilde{\hbar} \partial_{k}\right)$ :

$$
\left[\frac{k^{3}}{8}+\tilde{\hbar}\left(k \partial_{k}+\frac{1}{2}\right)\right] \psi(k)=\tilde{E} \psi(k) .
$$

This may be solved by using the ansatz $\psi(k)=A(k) \exp [i \Phi(k)]$ to give

$$
\psi(k)=\exp \left[-\frac{1}{2} \ln k+i \tilde{\hbar}^{-1}\left(-\tilde{E} \ln k+k^{3} / 24\right)\right] .
$$

The wave function in $\tilde{x}$ space is, consequently,

$$
\begin{aligned}
\psi(\tilde{x}) & =\int_{\mathcal{C}} d k \exp \left[-\frac{1}{2} \ln k+i \tilde{\hbar}^{-1}\left(k \tilde{x}-\tilde{E} \ln k+k^{3} / 24\right)\right] \\
& =\int_{\mathcal{C}} d k \exp \left[-\frac{1}{2} \ln k+i \tilde{\hbar}^{-1}\left(k \tilde{x}-\int \tilde{x}(k) d k\right)\right] .
\end{aligned}
$$

In the stationary phase (semiclassical WKB) approximation of $\tilde{E} \gg \tilde{\hbar}$, the integral (31) will be dominated by three saddle point solutions, which are the three roots $k(\tilde{E})$ of (24) for given $\tilde{E}$. Further on, we consider $\tilde{E}>0$. For given positive $\tilde{x}$ (outside the horizon), there is only one real solution, $k_{+s}$ (we use the notation of 6 ). For negative $\tilde{x}$ (inside the horizon) there are three such solutions, $k_{-s}, k_{+}$and $k_{-}$, provided that $\tilde{x}$ is not too small (cf. Fig. (1). The additional ingoing branch in this Figure (dotted line), close to the $x$ axis, is obtained if the full initial energy spectrum of (20) is used. This branch has comparatively high energy for given momentum and hence is not relevant for the present discussion. 
Far enough from the horizon, i.e. at $1 \gg \tilde{x} \gg \tilde{E}^{2 / 3}$, we have from (24) $k_{ \pm s} \simeq \tilde{E} / \tilde{x}$, and $k_{ \pm} \simeq \pm 2 \sqrt{2|\tilde{x}|}$. The branches $k_{ \pm s}=\tilde{E} / \tilde{x}$ exist in the linear dispersion case. At the horizon they approach $-\infty$ and $+\infty$, respectively. Nonlinear corrections to the energy spectrum become important when the momentum scale $\tilde{E}^{1 / 3}$ is reached, and these low momentum branches match with the high energy branches $k_{ \pm}$, which exist due to nonlinearity of the spectrum. The choice of the path $\mathcal{C}$ in the complex $k$ plane, determining the wave function, was discussed in detail int.

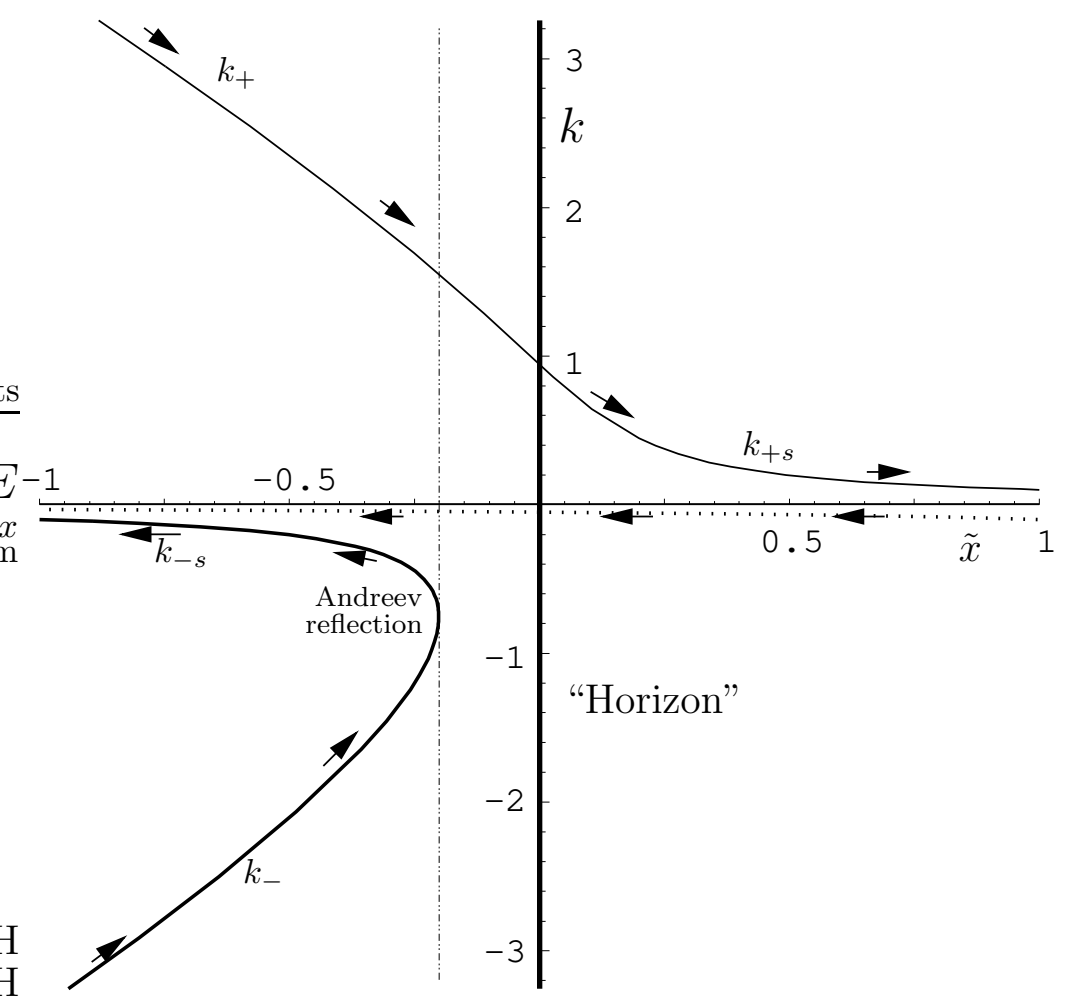

Figure 4: Quasiparticle trajectories in the flow $v_{\mathrm{s}}=-1+\tilde{x}$, for $\tilde{E}=0.1$. The notations are the same as those ind: $k_{ \pm}(x)$ are part of the branches with large positive and negative momenta and $k_{ \pm s}$ are part of the branches with small positive and negative momenta. The arrows indicate the direction of the laboratory frame group velocity of the quasiparticle. The horizon is identical with the $k$ axis. Thick lines for the quasiparticle trajectory show that the states with momenta $k_{-s}$ and $k_{-}$ are occupied in the comoving (free fall) vacuum. The dash-dotted line indicates the closest approach distance of the branch of large negative momenta to the horizon, at which the negative momentum $k_{-}$part of the wave coming from negative $\tilde{x}$ gets Andreev reflected (and the positive momentum $k_{+s}$ part is transmitted). The ingoing branch of very small negative momenta is shown by the dotted line. 
Depletion of the false vacuum occurs by tunneling from the trajectory $k_{-} \cdots k_{-s}$ to the outgoing mode trajectory $k_{+} \cdots k_{+s}$. In the WKB limit of $2 \pi \tilde{E} / \tilde{\hbar} \gg 1$, the high energy mode $k_{-}$is Andreev reflected 20 to $k_{-s}$, with a small probability of transmission to the outgoing mode. The transmission coefficient is exponentially small and corresponds to thermal radiation at the Hawking temperature:

$$
\mathcal{T} \simeq \exp [-2 \pi \tilde{E} / \tilde{\hbar}]=\exp \left[-E / T_{\mathrm{H}}\right]
$$

where the Hawking temperature is given by the equality $\tilde{T}_{\mathrm{H}}=\tilde{\hbar} / 2 \pi$. The Hawking temperature establishes a characteristic scale for the laboratory energy $\tilde{E} \sim \tilde{T}_{\mathrm{H}}$ (or $\left.E \sim T_{\mathrm{H}}\right)$ and thus the scales for $p, E^{\mathrm{com}}$ in $(25)$ and for the thickness of the shell $x_{0}=\left|x-x_{h}\right|$ in $(26)$. In dimensionless units one has $k \sim \tilde{E}^{\mathrm{com}} \sim \tilde{T}_{\mathrm{H}}^{1 / 3}, \tilde{x}_{0} \sim \tilde{T}_{\mathrm{H}}^{2 / 3}$.

The process of Andreev reflection occurs inside the horizon for our superluminal dispersion, whereas it is situated outside for subluminal dispersion 9 . In both cases the Hawking temperature does not depend on the dispersion. The point where the wave packet is turning back in co-ordinate space occurs where $d \tilde{x} / d k=0$, which is given by solving the equation $k_{0}^{3} / 4=-\tilde{E} \sqrt{1+k_{0}^{2} / 4}$. For small $k_{0}$, there results a value $k_{0} \simeq-(4 \tilde{E})^{1 / 3}=-1.59 \tilde{E}^{1 / 3}$, implying $\tilde{x}_{0}=\tilde{x}\left(k_{0}\right)=-\left(4^{-1 / 3}+4^{2 / 3} / 8\right) \tilde{E}^{2 / 3}=$ $-0.945 \tilde{E}^{2 / 3}$.

\subsection{Nonlinear velocity profiles}

An asymptotic form of the velocity profile $v_{\mathrm{s}}=f(\tilde{x})$, where $f(\tilde{x})$ is some function approaching zero (or a constant) at large positive $\tilde{x}$, and linearity at small $\delta \tilde{x}$ around the horizon, e.g. $f(\tilde{x})=-1+\tanh [\delta \tilde{x}])$, gives us the location of the quasiparticle in terms of its wave number as

$$
\tilde{x}(k)=f^{-1}\left(\tilde{E} / k-\sqrt{1+k^{2} / 4}\right) .
$$

We then obtain a Hamiltonian

$$
\tilde{H}=\tilde{E}^{\mathrm{com}}(k)+\{k f(\tilde{x})\},
$$

the curly brackets specifying that the appropriate operator ordering be taken for the expression contained within.

The influence of a particularly interesting flow profile on quasiparticle motion is depicted in Fig.5. The profile is uni-directional in the negative $x$ direction, and is developing locally superluminal velocities in a depression of the (symmetric) profile. It creates a characteristic combination of white hole and black hole (cf. 10 . tes for ${ }^{3} \mathrm{He}-\mathrm{A}$ and 18 for a Bose condensate). Specifically, there are bound quasiparticle states inside the horizon, which occur because of the combined effects of Andreev reflection, caused by nonlinear dispersion, and the shape of the velocity profile. 


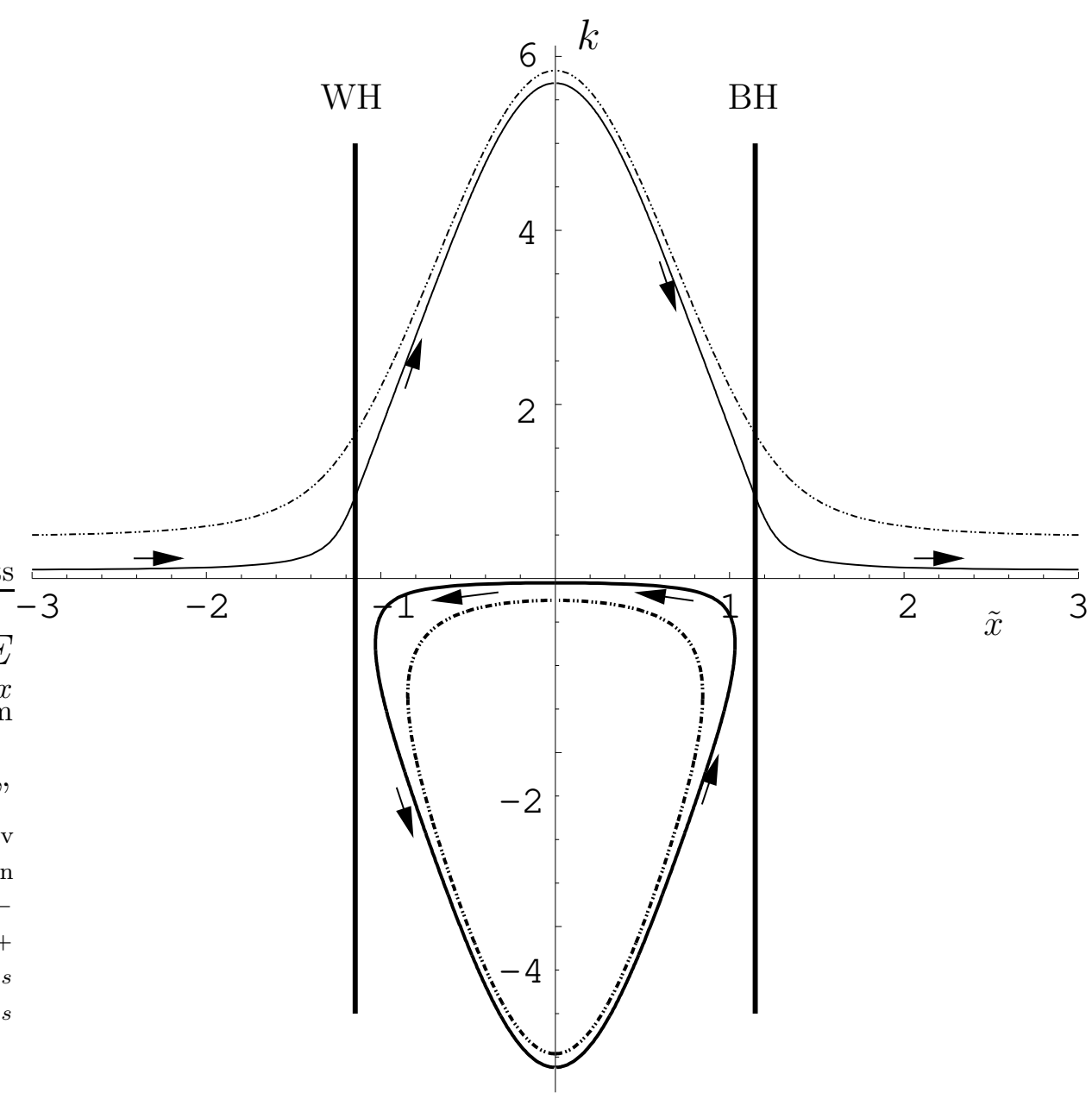

Figure 5: Large momentum quasiparticle phase space trajectories for the two (fixed) energies $\tilde{E}=0.1$ and $\tilde{E}=0.5$ (dashed), in the velocity profile $v_{\mathrm{s}}=$ $-\beta / \cosh ^{2}[\tilde{x} / \alpha](\beta>1)$, with $\alpha=1, \beta=3$. The black and white hole horizons where $v_{\mathrm{s}}=-1$ are located at $\tilde{x}_{h}= \pm \alpha \cosh ^{-1}(\sqrt{\beta})$, the upper (lower) sign valid for the black hole $(\mathrm{BH})$ and white hole $(\mathrm{WH})$, respectively. The horizon surface velocity gradient $\kappa_{\mathrm{s}}= \pm(2 / \alpha) \sqrt{1-\beta^{-1}}$. The quasiparticle states for negative momenta are bound states, where the values of the turning points at large $k$ are in leading order $k_{t}= \pm 2 \beta$ for large $\beta$.

\section{Dynamics of the normal component ("matter")}

Now we consider the classical temperature region $T \gg T_{\mathrm{H}}$, where the quantum processes of Hawking radiation can be neglected. Still, the behaviour of matter in the presence of the horizon is nontrivial and its description requires deviations from 
Lorentz invariance due to the blueshift in the vicinity of the horizon.

\section{1. "Relativistic" energy-momentum tensor of two fluid hydrodynamics}

The basic conservation equations of standard nonrelativistic Landau two fluid hydrodynamics (as expounded in detail in 12 $\S 8$ ), are those of total momentum and energy conservation. Energy and momentum can be exchanged between the two subsystems of quasiparticles and superfluid vacuum in a way similar to the exchange of energy and momentum between matter and the gravitational field. Moreover, in the low energy corner this exchange can be described in general relativistic form. The energy-momentum tensor derived from standard conservation equations of two fluid hydrodynamics 12 can be represented as 21 :

$$
\sqrt{-g} T_{\nu}^{\mu}=\sum_{\mathbf{p}} f v_{g}^{\mu} p_{\nu}, \quad v_{g}^{\mu} v_{g \mu}=-1+\frac{1}{c^{2}} \frac{\partial E^{\mathrm{com}}}{\partial p_{i}} \frac{\partial E^{\mathrm{com}}}{\partial p_{i}}
$$

where $f$ is the quasiparticle distribution function and $\sum_{\mathbf{p}} \equiv \sum_{s} \int d^{D} p /(2 \pi \hbar)^{D}$ designates summation over spin as well as momentum. The group four-velocity is defined as

$$
\begin{aligned}
v_{g}^{i} & =\frac{\partial E}{\partial p_{i}}, \quad v_{g}^{0}=1, \quad v_{g i}=\frac{1}{c^{2}} \frac{\partial E^{\mathrm{com}}}{\partial p_{i}}=\frac{1}{c^{2}}\left(v_{g}^{\mathrm{com}}\right)^{i}, \\
v_{g 0} & =-\left(1+\frac{1}{c^{2}} v_{\mathrm{s}}^{i} \frac{\partial E^{\mathrm{com}}}{\partial p_{i}}\right) .
\end{aligned}
$$

Space-Time indices $\mu, \nu, \ldots$ are throughout assumed to be raised and lowered by the Painlevé-Gullstrand metric. The group four-velocity is null in the relativistic domain of the spectrum only: $v_{g}^{\mu} v_{g \mu}=0$ if $E^{\text {com }}=p$. Here and in what follows, we again set $c=c_{\perp}=1$ (scale velocities with $c$ ), except where the "speed of light" is explicitly indicated for clarity.

The energy-momentum tensor thus defined satisfies the covariant conservation law equation

$$
T_{\nu ; \mu}^{\mu}=\frac{1}{\sqrt{-g}} \frac{\partial\left(T_{\nu}^{\mu} \sqrt{-g}\right)}{\partial x^{\mu}}-\frac{1}{2} \frac{\partial g_{\alpha \beta}}{\partial x^{\mu}} T^{\alpha \beta}=0
$$

As pointed out by Landau and Lifshitz22, this equation does not generally express any conservation law whatever. The same is true for quasiparticles in superfluids. This equation does not mean that energy and momentum of quasiparticles are necessarily conserved: In the presence of an inhomogeneous condensate the second term describes the energy and momentum exchange between quasiparticles (matter) and superfluid vacuum (gravity field)

$$
\left(\sqrt{-g} T^{\mu}{ }_{\nu, \mu}=P_{i} \partial_{\nu} v_{\mathrm{s}}^{i}+\sum_{\mathbf{p}} f \partial_{\nu} E^{\mathrm{com}}\right.
$$

where $\mathbf{P}=\sum_{\mathbf{p}} \mathbf{p} f(\mathbf{r}, \mathbf{p})$ is the quasiparticle momentum density. The last term appears only if the "speed of light" depends on space-time. Further on we neglect this term. 
In a one-dimensional situation, and assuming that there is no temporal dependence of superfluid velocity, respectively if it is permitted that it is neglected, we obtain an energy conservation law for quasiparticles in the form

$$
\frac{\partial}{\partial t}\left(\sqrt{-g} T_{0}^{0}\right)+\frac{\partial}{\partial x}\left(\sqrt{-g} T_{0}^{x}\right)=0
$$

which tells us that in any time independent situation, the energy flux $\sqrt{-g} T^{x}{ }_{0}$ is necessarily constant in space. The momentum conservation in $x$ direction reads

$$
\frac{\partial}{\partial t}\left(\sqrt{-g} T^{0}{ }_{x}\right)+\frac{\partial}{\partial x}\left(\sqrt{-g} T_{x}^{x}\right)=-P_{x} \partial_{x} v_{\mathrm{s}}=-\frac{\kappa}{v_{\mathrm{s}}} T_{x}^{0} \sqrt{-g} .
$$

It contains the velocity gradient as a source term, which corresponds to the "gravity" $\kappa=\partial_{x}\left(v_{\mathrm{s}}^{2} / 2\right)$, where $v_{\mathrm{s}}^{2} / 2$ plays the role of the gravitational potential.

The relevant ${ }^{\ddagger}$ components of the energy-momentum tensor are $\left(p_{0}=-E, p^{0}=\right.$ $\left.E^{\mathrm{com}}\right)$ :

$$
\begin{aligned}
\sqrt{-g} T_{i}^{0} & =\sum_{\mathbf{p}} f p_{i}=P_{i} \quad \text { Momentum density in either frame, } \\
\sqrt{-g} T_{0}^{0} & =-\sum_{\mathbf{p}} f E \quad \text { (Negative) Laboratory frame energy density, } \\
\sqrt{-g} T^{k}{ }_{i} & =\sum_{\mathbf{p}} f p_{i} v_{g}^{k} \quad \text { Laboratory frame momentum flux, } \\
\sqrt{-g} T^{i}{ }_{0} & =-\sum_{\mathbf{p}} f E v_{g}^{i} \quad \text { (Negative) Laboratory frame energy flux, } \\
\sqrt{-g} T^{00} & =\sum_{\mathbf{p}} f p^{0}=\sum_{\mathbf{p}} f E^{\mathrm{com}} \quad \text { Comoving frame energy density. }
\end{aligned}
$$

We will further on keep the square root of the negative metric determinant for structural clarity, though it is a space-time constant in our considerations, as we assume the "speed of light" to be constant below. The relation between the laboratory frame energy density and that in the comoving frame expresses the underlying Galilean invariance:

$$
-\sqrt{-g} T_{0}^{0}=\sum_{\mathbf{p}} f E=\sqrt{-g} T^{00}+v_{\mathrm{s}}^{i} P_{i}
$$

The additional hydrodynamic equation to be satisfied is that of the conservation of the number of particles comprising the underlying liquid, say, the number of ${ }^{3} \mathrm{He}$ atoms. If temporal variations of the density are negligible, this amounts to requiring that the total momentum density

$$
\mathbf{j}=\rho \mathbf{v}_{\mathrm{s}}+\mathbf{P}
$$

¥ "Relevant" are termed those components of the energy-momentum tensor that do not depend on the low energy properties of the quasiparticles, i.e. on the form and existence of a Lorentz metric. 
has vanishing divergence, $\nabla \cdot \mathbf{j}=0$. That we can neglect (temporal and spatial) variations of the density will be true in the case of ${ }^{3} \mathrm{He}-\mathrm{A}$, where the "speed of light" $c_{\perp}$ is much smaller than the sound velocity. This is in marked contrast to truly "acoustic" black holes, as they will exhibit large variations of the density for velocities of the fluid above the speed of sound.

\subsection{Components of energy-momentum in local thermodynamic equilib- rium}

Local thermodynamic equilibrium is characterized by the local temperature $T$ and local velocity $\mathbf{v}_{n}$ of the quasiparticle system. The equilibrium distribution function is 12 .

$$
f_{\mathcal{T}}=\frac{1}{1+\exp \left[T^{-1}\left(E(\mathbf{p})-\mathbf{p} \cdot \mathbf{v}_{n}\right]\right.}=\frac{1}{1+\exp \left[T^{-1}\left(E^{\operatorname{com}}(\mathbf{p})-p_{i} w^{i}(x)\right)\right]} .
$$

where $\mathbf{w}=\mathbf{v}_{\mathrm{n}}-\mathbf{v}_{\mathrm{s}}$ is the so-called counterflow velocity.

In local thermodynamic equilibrium the components of energy-momentum for the quasiparticle system (matter) are determined by the generic thermodynamic potential (the pressure), which for fermions has the form

$$
\Omega=-T \frac{1}{(2 \pi \hbar)^{D}} \sum_{s} \int d^{D} p \ln (1-f) .
$$

The momentum density is expressed in terms of $\Omega$ as

$$
P_{i}=\sqrt{-g} T_{i}^{0}=\frac{1}{(2 \pi \hbar)^{D}} \sum_{s} \int d^{D} p f p_{i}=\frac{\partial \Omega}{\partial w^{i}} \equiv\left(\rho_{\mathrm{n}}\right)_{i j} w^{j} .
$$

Here $\rho_{n}$ is the so-called density of the normal component (in general a tensorial quantity), so that the total current of the liquid: $\mathbf{j}=\rho \mathbf{v}_{\mathbf{s}}+\rho_{\mathrm{n}}\left(\mathbf{v}_{\mathrm{n}}-\mathbf{v}_{\mathrm{s}}\right) \equiv \rho_{\mathrm{s}} \mathbf{v}_{\mathrm{s}}+\rho_{\mathrm{n}} \mathbf{v}_{\mathrm{n}}$, where $\rho_{\mathrm{s}}=\rho-\rho_{\mathrm{n}}$ is the density of the superfluid component.

Considering below for simplicity the (spatially) one-dimensional case $(D=1)$, one has for the purely "relativistic" contribution such that $E^{\mathrm{com}}=\left|p_{x}\right|$ (and thus $\left.\left(v_{g}^{\mathrm{com}}\right)_{x}=1\right)$ :

$$
\begin{gathered}
\Omega=\sum_{s} \frac{\pi}{12 \hbar} T_{\text {eff }}^{2}, \quad T_{\text {eff }}=\frac{T}{\sqrt{1-w^{2}}}, \\
P_{x}=\sqrt{-g} T^{0}{ }_{x}=\rho_{\mathrm{n}} w, \quad \rho_{\mathrm{n}}=\frac{2 \Omega}{1-w^{2}} .
\end{gathered}
$$

The other components are in the "relativistic" case then given as

$$
T^{\mu \nu}=(\varepsilon+\Omega) u^{\mu} u^{\nu}+\Omega g^{\mu \nu}, \quad \varepsilon=-\Omega+T \frac{\partial \Omega}{\partial T}=D \Omega, \quad T_{\mu}^{\mu}=0 .
$$

where $u^{\alpha}$ and $u_{\alpha}=g_{\alpha \beta} u^{\beta}$, which satisfy the normalisation equation $u_{\alpha} u^{\alpha}=-1$, are

$$
u^{0}=\frac{1}{\sqrt{1-w^{2}}}, \quad u^{i}=\frac{v_{(n)}^{i}}{\sqrt{1-w^{2}}}, \quad u_{i}=\frac{w_{i}}{\sqrt{1-w^{2}}}, \quad u_{0}=-\frac{1+\mathbf{w} \cdot \mathbf{v}_{(s)}}{\sqrt{1-w^{2}}},
$$


The distribution of quasiparticles in local equilibrium in (44) is expressed via the temperature four-vector $\beta^{\mu}$ :

$$
f_{\mathcal{T}}=\frac{1}{1+\exp \left[-\beta^{\mu} p_{\mu}\right]}, \quad \beta^{\mu}=\frac{u^{\mu}}{T_{\mathrm{eff}}}=\left(\frac{1}{T}, \frac{\mathbf{v}_{n}}{T}\right), \quad \beta^{\mu} \beta_{\mu}=-T_{\mathrm{eff}}^{-2} .
$$

For the relativistic system, true equilibrium with vanishing entropy production is established if $\beta^{\mu}$ is a timelike Killing vector:

$$
\beta_{\mu ; \nu}+\beta_{\nu ; \mu}=0, \text { or } \quad \beta^{\alpha} \partial_{\alpha} g_{\mu \nu}+\left(g_{\mu \alpha} \partial_{\nu}+g_{\nu \alpha} \partial_{\mu}\right) \beta^{\alpha}=0 .
$$

For a time independent, space dependent situation the condition $0=\beta_{0 ; 0}=\beta^{i} \partial_{i} g_{00}$ gives $\beta^{i}=0$, while the other conditions are satisfied when $\beta^{0}=$ constant. Hence true equilibrium requires that $\mathbf{v}_{n}=0$ in the frame of the velocity texture, and $T=$ constant. These are just the equilibrium conditions in superfluids. They correspond to Tolman's law in general relativity, with Tolman temperature being equal to the real temperature of the liquid:

$$
T_{\text {eff }}=\frac{T}{\sqrt{1-v_{\mathrm{s}}^{2}}}=\frac{T}{\sqrt{-g_{00}}} .
$$

\section{Thermal states in the presence of a horizon}

\subsection{One-dimensional hydrodynamic equations}

To describe the thermal states in the presence of the horizon in a most simple way, we consider a purely one-dimensional situation. This is, however, not completely unrealistic an assumption. Of course, we have to relax the condition $j=$ const., which does not allow for a velocity $v_{\mathrm{s}}$ gradient in the one-dimensional case. This, however, can be achieved by the inhomogeneous profile of a tube. Then there is some flow in the transverse directions, such that $\nabla \cdot \mathbf{j}=0$ is satisfied. An alternative possibility to construct a one-dimensional horizon is to make $c_{\perp}(x)$ space dependent 10 . The mass conservation law $\mathbf{j}=$ const. also implies that the nonzero quasiparticle momentum, which arises, say, due to Hawking radiation, has to be compensated by a corresponding change of the superflow velocity. This is the mechanism of back-reaction of the superfluid vacuum to quasiparticle dynamics. Back-reaction is, however, small and can be neglected, namely if the effective temperature of quasiparticles is small compared to the superfluid transition temperature $T_{c}$. This will always be true in our approximation, for which $T<m_{*} c_{\perp}^{2} \ll T_{c}$. Then, $P \ll \rho v_{\mathrm{s}}$.

We will investigate below a case for which the quantum effects related to gravity, including the Hawking radiation process, can be neglected. This is permitted if all the relevant energies are much higher than the Hawking temperature: $T \gg T_{\mathrm{H}}$, and $\hbar / \tau \gg T_{\mathrm{H}}$. In the latter relation, $l=c \tau$ is the mean free path, and the relation means that $l$ is small compared with the characteristic length, within which the 
velocity (the "gravitational potential") changes: $l\left(\partial v_{\mathrm{s}} / \partial x\right) \ll 1$. This is just the condition for the applicability of the two fluid hydrodynamic equations, where the variables are the superfluid velocity $v_{\mathrm{s}}$ which, when squared, plays the part of the gravitational potential, as well as temperature $T(x)$ and velocity $v_{\mathrm{n}}(x)$ of the normal component, which characterize the local equilibrium states of quasiparticles (matter). The dissipative terms in the two fluid equations can then be neglected in a zeroth order approximation, since they are small compared to the reversibly hydrodynamic terms by the above parameter $l\left(\partial v_{\mathrm{s}} / \partial x\right) \ll 1$.

If the superfluid velocity (gravity) field is fixed, the other hydrodynamic variables, temperature $T(x)$ and velocity $v_{\mathrm{n}}(x)$ of "matter", are determined by the conservation of energy and momentum. From equation (39) it follows that the energy flux $Q$ carried by the quasiparticles is constant. In the relativistic approximation one then has

$$
Q=-\sqrt{-g} T^{x}{ }_{0}=2 \Omega \frac{v_{\mathrm{n}}\left(1+w v_{\mathrm{s}}\right)}{1-w^{2}}=\mathrm{const}, \quad \Omega=\frac{(2 s+1) \pi}{12} T_{\mathrm{eff}}^{2} .
$$

From (40) there results the first order differential equation

$$
-\partial_{x}\left(2 \Omega \frac{v_{\mathrm{n}} w}{1-w^{2}}+\Omega\right)=2 \Omega \frac{w}{1-w^{2}} \partial_{x} v_{\mathrm{s}} .
$$

\subsection{Local equilibrium states with zero energy flux}

In the case that the energy flux is zero, equation (54) yields two possible states.

\subsubsection{True equilibrium outside horizon}

Equation (54) is satisfied by the trivial solution $v_{\mathrm{n}}=0$. Then from (55) it follows that $T=$ constant. This corresponds to a true equilibrium state outside the horizon. The effective temperature satisfies Tolman's law in (53): $T_{\text {eff }}(x)=$ $T / \sqrt{-g_{00}(x)}=T / \sqrt{1-v_{\mathrm{s}}^{2}(x)}$. This equilibrium state cannot be continued across the horizon: The effective temperature $T_{\text {eff }}$ diverges when the horizon is approached and becomes imaginary inside the horizon, where $|w|=\left|v_{\mathrm{s}}\right|>1$.

\subsubsection{Thermal state behind horizon}

Equation (54) is satisfied by $1+w v_{\mathrm{s}}=0$ (or $u_{0}=0$ ). Since $w^{2}<1$, this solution can be valid only inside the horizon, where $v_{\mathrm{s}}^{2}>1$. From (55) it follows that $\Omega=\mathrm{const} /\left(v_{\mathrm{s}}^{2}-1\right)$, and thus the temperature behaves as $T^{2} \propto \Omega\left(1-w^{2}\right)=$ const $/ v_{\mathrm{s}}^{2}$, or $T=T_{\text {hor, left }} /\left|v_{\mathrm{s}}\right|$, where $T_{\text {hor, left }}$ is the temperature at the horizon when approached from inside. Thus inside the horizon one has a quasi-equilibrium state with inhomogeneous temperature. The effective temperature behind the horizon follows a modified Tolman law:

$$
T_{\text {eff }}=\frac{T_{\text {hor,left }}}{\sqrt{v_{\mathrm{s}}^{2}(x)-1}}
$$


The above equation is valid only in $1+1$ dimensions. For larger spatial dimension $D>1$ one has $\Omega \propto\left(v_{\mathrm{s}}^{2}-1\right)^{-(D+1) / 2 D}$ and $T_{\text {eff }} \propto\left(v_{\mathrm{s}}^{2}-1\right)^{-1 / 2 D}$, so that $T \propto$ $\left(v_{\mathrm{s}}^{2}-1\right)^{(D-1) / 2 D} /\left|v_{\mathrm{s}}\right|$.

The thermal state behind the horizon is no longer determined by the Hamiltonian $H(x>0)=-p_{0}=E$, but can be described as a local equilibrium state if one uses the kinetic momentum as the Hamiltonian:

$$
\begin{array}{r}
f(p, x<0)=\frac{1}{1+\exp \left(H(x<0) / T_{\text {hor,left }}\right)}, \\
H(x<0)=-p^{1}, \quad p^{1}=\left(1-v_{\mathrm{s}}^{2}\right) p+v_{\mathrm{s}} E=p+v_{\mathrm{s}}|p|=-p^{0} \dot{x} .
\end{array}
$$

Here $\dot{x}=d E / d p$, so that $p^{1}$ is a "mass" multiplied by a velocity and thus represents a kinetic momentum. The reason why the kinetic momentum plays the part of the Hamiltonian is that

$$
\frac{p^{1}}{T_{\text {hor,left }}}=\beta^{\mu}(x<0) p_{\mu}, \quad \beta^{\mu}=\left(\frac{v_{\mathrm{s}}}{T_{\text {hor,left }}}, \frac{1-v_{\mathrm{s}}^{2}}{T_{\text {hor, left }}}\right) .
$$

Here $\beta^{\mu}(x<0)$ is a two-temperature, which satisfies the CKV condition $\beta_{\mu ; \nu}+$ $\beta_{\nu ; \mu} \propto g_{\mu \nu}$. It can be verified by direct calculation that

$$
\beta_{\mu ; \nu}+\beta_{\nu ; \mu}=g_{\mu \nu} \partial_{x}\left(1-v_{\mathrm{s}}^{2}\right) .
$$

For massless relativistic particles the CKV condition is sufficient for thermal equilibrium states without dissipation to exist. Thus, despite the inhomogeneous temperature and nonzero normal velocity $v_{\mathrm{n}}$, the state behind the horizon becomes a true equilibrium state in the low energy relativistic limit.

Existence of nondissipative thermal states behind the horizon is a consequence of the 1+1-dimensional situation, in which the metric is conformally flat. The coordinate transformation $\hat{t}=t-\int^{x} d x^{\prime} v_{\mathrm{s}} /\left(1-v_{\mathrm{s}}^{2}\right), \hat{x}=x$ transforms the vector $\beta^{\mu} \rightarrow \hat{\beta}^{\mu}=\left(0,1-v_{\mathrm{s}}^{2}\right)$, so that $\hat{p}^{1}=-i\left(1-v_{\mathrm{s}}^{2}\right) \partial_{\hat{x}}$. Another coordinate transformation $d x /\left(1-v_{\mathrm{s}}^{2}\right)=d \hat{x}$ transforms $p^{1}$ to $-i \partial_{\hat{x}}$. On the other hand, these two transformations give the conformally flat metric $d \hat{s}^{2}=\left(1-v_{\mathrm{s}}^{2}\right)\left(-d t^{2}+d x^{2}\right)$ with the conformal factor $1-v_{\mathrm{s}}^{2}$. This implies that $p^{1}$ is a conformal Killing vector satisfying the CKV condition in (60).

\subsubsection{Matching thermal states across horizon}

Since the superluminal dispersion provides an energy exchange between the matter (quasiparticles) inside and outside the horizon, the temperature must be continuous across the horizon. Thus $T_{\text {hor,left }}=T_{\text {hor,right }}$. It is important to note that even in the comoving frame, the blueshifted energies are finite and much smaller than the "Planck" scale, on which the hydrodynamic formalism breaks down. Consequently, the temperature and the normal fluid velocity can be well determined not only on both sides of the horizon but even at the horizon itself, where all the 
characteristic scales are determined by the first nonlinear correction to the quasiparticle spectrum. In our $1+1 \mathrm{~d}$ case thermal states on both sides of the horizon do, however, not depend on the details of this thin layer. The only effective output of the membrane is that it establishes the boundary condition $T_{\text {hor, left }}=T_{\text {hor,right }}$.

Thus, assuming that at $+\infty$ the superfluid velocity vanishes, one has the following temperature profile on both sides of the horizon: $T(x>0)=T_{\infty}, T(x<0)=$ $T_{\infty} /\left|v_{\mathrm{s}}(x)\right|$. The distribution functions on both sides of the horizon have the form

$$
\begin{array}{ll}
f(p, x>0)=\frac{1}{1+\exp \left(-p_{0} / T_{\infty}\right)}, & p_{0}=|p|+v_{\mathrm{s}} p, \\
f(p, x<0)=\frac{1}{1+\exp \left(-p^{1} / T_{\infty}\right)}, & p^{1}=v_{\mathrm{s}}|p|+p .
\end{array}
$$

There is a symmetry between the states inside and outside the horizon. While outside the horizon one has Tolman's law, $T(x>0)=T_{\text {eff }} \sqrt{1-v_{\mathrm{s}}^{2}}$, the temperature behind the horizon is obtained from Tolman's law by the substitution $v_{\mathrm{s}} \rightarrow 1 / v_{\mathrm{s}}$, i.e., $T(x<0)=T_{\text {eff }} \sqrt{1-1 / v_{\mathrm{s}}^{2}}$. The same procedure applies to $w$ : One has $w=-v_{\mathrm{s}}$ outside the horizon and $w=-1 / v_{\mathrm{s}}$ inside. The temperature two-vector for the state behind horizon

$$
\beta^{\mu}(x<0)=\left(\beta^{0}, \beta^{1}\right)=\frac{1}{T_{\infty}}\left(-v_{\mathrm{s}}, 1-v_{\mathrm{s}}^{2}\right), \quad \beta_{\mu}(x<0)=\left(\beta_{0}, \beta_{1}\right)=\frac{1}{T_{\infty}}(0,1),
$$

can be compared with the temperature two-vector for the true equilibrium outside the horizon

$$
\beta^{\mu}(x>0)=\frac{1}{T_{\infty}}(1,0), \quad \beta_{\mu}(x>0)=\frac{1}{T_{\infty}}\left(v_{\mathrm{s}}^{2}-1,-v_{\mathrm{s}}\right) .
$$

The relation between these two-temperatures shows that the states across the horizon are dual to each other:

$$
\beta^{\mu}(x>0)=\epsilon^{\mu \nu} \beta_{\mu}(x<0),
$$

i.e., the space and time components of the two-temperature transform to each other across the horizon $\left(\epsilon^{11}=\epsilon^{22}=0, \epsilon^{12}=-\epsilon^{21}=1\right)$. It also appears that the thermal equilibrium behind the horizon is the same as the thermal equilibrium outside the horizon, if the superfluid velocity and the speed of light are interchanged. Behind the horizon the speed of light plays the part of the superfluid velocity and vice versa. This corresponds to the case of a soliton moving in superfluid ${ }^{3} \mathrm{He}-\mathrm{A}$, where the speed of light changes in space, while the superfluid velocity remains constant 10 .

\subsection{Local equilibrium states with nonzero energy flux}

If the energy flux is nonzero, we express $\Omega\left(v_{\mathrm{s}}, w\right)=Q\left(1-w^{2}\right) / 2 v_{\mathrm{n}}\left(1+w v_{\mathrm{s}}\right)$ using (54), and substitute it into (55). One obtains an equation for $w\left(v_{\mathrm{s}}\right)$ :

$$
\frac{\partial w}{\partial v_{\mathrm{s}}}=-\frac{1-w^{2}}{1-v_{\mathrm{s}}^{2}}
$$


which has the solution

$$
w=\frac{\left(1-v_{\mathrm{s}}\right)-C\left(1+v_{\mathrm{s}}\right)}{\left(1-v_{\mathrm{s}}\right)+C\left(1+v_{\mathrm{s}}\right)}
$$

where $C$ is a constant. Using this equation we have the sequence of relations:

$$
\begin{aligned}
v_{\mathrm{n}} & =w+v_{\mathrm{s}}=(1-C) \frac{1-v_{\mathrm{s}}^{2}}{\left(1-v_{\mathrm{s}}\right)+C\left(1+v_{\mathrm{s}}\right)}, \\
1+w v_{\mathrm{s}} & =(1+C) \frac{1-v_{\mathrm{s}}^{2}}{\left(1-v_{\mathrm{s}}\right)+C\left(1+v_{\mathrm{s}}\right)}, \\
\frac{v_{\mathrm{n}}\left(1+w v_{\mathrm{s}}\right)}{1-w^{2}} & =\left(1-v_{\mathrm{s}}^{2}\right) \frac{1-C^{2}}{4 C} .
\end{aligned}
$$

Since $|w| \leq 1$, one has $C_{+}>0$ outside the horizon, where $\left|v_{\mathrm{s}}\right|<1$, and $C_{-}<0$ inside the horizon, where $\left|v_{\mathrm{s}}\right|>1$. The energy flux is

$$
Q=2 \frac{(2 s+1) \pi}{12} \frac{1-C^{2}}{4 C}\left(1-v_{\mathrm{s}}^{2}\right) T_{\mathrm{eff}}^{2} .
$$

Since the energy flux can be only inward, $Q<0$, (in the absence of Hawking radiation), one has $C^{2}>1$. From (69) it follows that the effective temperature exhibits Tolman's law ${ }^{\S}$, determined by the temperature at $+\infty$ (if $v_{\mathrm{s}}(+\infty)=0$ is assumed):

$$
T_{\text {eff }}=\frac{T_{\infty}}{\sqrt{\left|1-v_{\mathrm{s}}^{2}\right|}}, \quad T_{\infty}^{2}=|Q| \frac{24}{(2 s+1) \pi} \frac{|C|}{C^{2}-1} .
$$

It follows from the above relation that a modified form of Tolman's law, telling us that the "temperature" detected by a local observer, $T^{\text {eff }}=$ const. $/ \sqrt{\left|-g_{00}\right|}=$ const. $/ \sqrt{\left|1-v_{\mathrm{s}}^{2}\right|}$, is fulfilled even in the absence of complete equilibrium. "Temperature" is set in quotation marks, because $T^{\text {eff }}$ represents only an effective "relativistic" temperature of the quasiparticles appearing in the distribution function, while the true temperature of the superfluid still remains $T$. The effective temperature diverges at the horizon, while the true temperature remains finite, but changes in space:

$$
T=\sqrt{1-w^{2}} T_{\mathrm{eff}}=T_{\infty} \frac{\sqrt{1-w^{2}}}{\sqrt{\left|1-v_{\mathrm{s}}^{2}\right|}}=T_{\infty} \frac{2 \sqrt{|C|}}{\left|1-v_{\mathrm{s}}+C\left(1+v_{\mathrm{s}}\right)\right|}
$$

and approaches at the horizon the value

$$
T_{\text {hor }}=T_{\infty} \sqrt{|C|}
$$

Since the true temperature must be continuous across the horizon, the condition $C_{-}=-C_{+}$is enforced.

\footnotetext{
$\S$ The relativistic Tolman law of a Lorentzian space-time obviously involves corrections from the
} nonrelativistic tail. These corrections are, however, small far enough away from the horizon. 
Hence the continuity of $T$ and the conservation of the energy flux completely determine the thermal state inside the horizon in terms of the temperature at positive infinity, $T_{\infty}$, and the energy flux $Q$. In terms of $T_{\infty}$ and $v_{\mathrm{n}}(+\infty)$, the relations $v_{\mathrm{n}}(+\infty)=\left(1-C_{+}\right) /\left(1+C_{+}\right)$or $C_{+}=\left(1-v_{\mathrm{n}}(+\infty)\right) /\left(1+v_{\mathrm{n}}(+\infty)\right)$ hold. In the limit of vanishing flux, $|C| \rightarrow 1$, one obtains the results of the previous subsection:

$$
\begin{array}{r}
\text { outside horizon : } \quad T=T_{\infty}, v_{\mathrm{n}}=0, w(x)=-v_{\mathrm{s}}(x), \\
\text { inside horizon : } \quad T(x)=\frac{T_{\infty}}{\left|v_{\mathrm{s}}(x)\right|}, v_{\mathrm{n}}(x)=\frac{v_{\mathrm{s}}^{2}(x)-1}{v_{\mathrm{s}}(x)}, w(x)=-\frac{1}{v_{\mathrm{s}}(x)} .
\end{array}
$$

\section{Properties of the horizon}

\subsection{Kinks at the horizon}

In the presence of superluminal dispersion, all physical quantities are continuous at the horizon. On the other hand, the thickness of the membrane, determined by the nonlinear disperion in $(26)$ with $E=T_{\infty}$, is very small and can be neglected. In this limit the thermal quantities experience kinks. For example, $\left.\nabla T\right|_{+0}=\left.\nabla T\right|_{-0}\left(C_{+}-1\right) /\left(C_{-}-1\right) ;\left.\nabla w\right|_{ \pm 0}=-C_{ \pm} \nabla v_{\mathrm{s}}(x=0)$. In the case of zero flux, equations $(73 \sqrt{74})$, the jump in the derivative of the temperature is $\left.\nabla T\right|_{+0}-\left.\nabla T\right|_{-0}=2 \pi T_{\infty} T_{\mathrm{H}}$, and does not depend on details of the dispersion. This means that in the limit of a purely relativistic system, the presence of a nonzero temperature at infinity implies a singularity at the horizon. This singularity at the horizon cannot be removed, since in the presence of "matter" with nonzero temperature the system is not invariant under coordinate transformations, and this produces a kink in temperature at the horizon.

\subsection{Entropy related to horizon}

The thermal states also have an entropy profile, for which we can distinguish the part coming from the bulk and that from the horizon. Typically the entropy related to the horizon is dominating, since it involves the "Planck" scale parameter. We again discuss the $1+1 \mathrm{~d}$ case, where the horizon contribution is only logarithmically larger than the contribution of the bulk.

The true entropy of superfluid, which is carried by its normal subsystem at $T_{\infty} \gg T_{\mathrm{H}}$, is

$$
\mathcal{S}=\int d^{D} r S, \quad S=\frac{\partial \Omega}{\partial T} .
$$

The "relativistic" entropy, which is measured by a local observer living in the quasiparticle world is the effective entropy

$$
S_{\mathrm{eff}}=\frac{\partial \Omega}{\partial T_{\mathrm{eff}}}=\frac{\partial \Omega}{\partial T} \frac{\partial T}{\partial T_{\mathrm{eff}}}=S \sqrt{1-w^{2}} .
$$


In the presence of a horizon, the total true entropy can be divided into 3 contributions:

$$
\mathcal{S}=\mathcal{S}_{\text {ext }}+\mathcal{S}_{\text {int }}+\mathcal{S}_{\text {hor }}
$$

The exterior entropy, which comes from the bulk liquid, is proportional to the size $L_{\text {ext }}$ of the external region: $\mathcal{S}_{\text {ext }} \propto T_{\infty} L_{\text {ext }}$. A similar estimate holds for the entropy of the interior region, $\mathcal{S}_{\text {int }} \propto T_{\infty} L_{\text {int }}$. The entropy related to the horizon stems from the logarithmically divergent contribution at the horizon:

$$
\mathcal{S}_{\mathrm{hor}}=\frac{(2 s+1) \pi}{6}\left(\int_{-1 / \kappa_{\mathrm{s}}}^{-x_{0}}+\int_{x_{0}}^{1 / \kappa_{\mathrm{s}}}\right) d x \frac{T_{\infty}}{\left|1-v_{\mathrm{s}}^{2}\right|}, \quad 1+v_{\mathrm{s}} \approx \kappa_{\mathrm{s}} x,
$$

where we have used relations (73) and (74) (valid for the limes of vanishing energy flux). According to (24), the ultraviolet cutoff $x_{0}$ is provided by the thickness of the shell (membrane) determined by the nonlinear dispersion, which gives (see equation (25) with $\left.E=T_{\infty}\right)$ :

$$
x_{0} \kappa_{\mathrm{S}}=\tilde{x}_{0} \sim \tilde{T}_{\infty}^{2 / 3}=\left(\frac{T_{\infty}}{E_{\text {Planck }}}\right)^{2 / 3}
$$

As a result one has

$$
\mathcal{S}_{\text {hor }}=\frac{(2 s+1)}{18} \frac{T_{\infty}}{T_{\mathrm{H}}} \ln \left(\frac{E_{\text {Planck }}}{T_{\infty}}\right) .
$$

This relation also means that the density of quasiparticle states diverges logarithmically at the horizon:

$$
\begin{gathered}
N_{\text {hor }}(E)=(2 s+1) \int \frac{d x d p}{2 \pi} \delta(E-E(p, x))=\frac{(2 s+1)}{6 \pi^{2} T_{\mathrm{H}}} \ln \left(\frac{E_{\text {Planck }}}{E}\right), \\
T_{\mathrm{H}} \ll E \ll E_{\text {Planck }}
\end{gathered}
$$

\subsection{Dissipation at the horizon}

Behind the horizon the conditions for the true equilibrium in (52) are not satisfied, $u_{\mu \nu} \equiv \beta_{\mu ; \nu}+\beta_{\nu ; \mu} \neq 0$, cf. equation (60):

$$
u_{00}=\frac{1}{T_{\infty}}\left(1-v_{\mathrm{s}}^{2}\right) \partial_{x}\left(v_{\mathrm{s}}^{2}\right), \quad u_{01}=\frac{v_{\mathrm{s}}}{T_{\infty}} \partial_{x}\left(v_{\mathrm{s}}^{2}\right), \quad u_{11}=-\frac{1}{T_{\infty}} \partial_{x}\left(v_{\mathrm{s}}^{2}\right) .
$$

But quasi-equilibrium CKV conditions are met, with the conformal function being equal to the local gravitational field, normalized to the temperature at infinity:

$$
u_{\mu \nu}=2 g_{\mu \nu} \Phi, \quad \Phi=-\frac{1}{T_{\infty}} \partial_{x}\left(\frac{g_{00}}{2}\right) .
$$

Since the true temperature and the normal component velocity, $T$ and $v_{\mathrm{n}}$, are space dependent, there is entropy production and dissipation stemming from thermal 
conductivity, giving a term $\frac{\kappa}{T}(\nabla T)^{2}$, and second viscosity, contributing a term $\zeta_{2}\left(\operatorname{div} \mathbf{v}_{\mathbf{n}}\right)^{2}$. In the truly relativistic régime with massless fermions dissipation is, however, absent since the CKV conditions are satisfied. This fact is also known for the bosonic case, superfluid ${ }^{4} \mathrm{He}$, where, according to Khalatnikov 12 , these two transport parameters are zero in a pure phonon gas. They become nonzero for any deviation from the linear phonon spectrum. Thus, if the Hawking process is neglected, in the purely relativistic régime, there will be global equilibrium states on both sides of the horizon, for any given temperature at infinity. Dissipation occurs only due to deviations from the relativistic spectrum. These deviations are most pronounced and effective within the thin shell of the horizon vicinity, so that the main source of dissipation in a $1+1 \mathrm{~d}$ superfluid, in the presence of a Landau horizon, will be concentrated at the membrane. The dissipation is determined by the gradient of the superfluid velocity at the horizon.

This, then, is the thermal counterpart of Hawking radiation. The latter becomes important only when the quantum limit is approached, i.e., when $T_{\infty}$ approaches the Hawking temperature $T_{\mathrm{H}}$. The hydrodynamic dissipation at $T_{\infty} \gg T_{\mathrm{H}}$ does the same job as Hawking radiation at $T=0$, leading to the final extinction of the black hole. In our case, this means deceleration of the superfluid below $\left|v_{\mathrm{s}}\right|=1$ and thus the consequent merging of black and white hole horizons (cf. Fig. E), and, finally, their mutual annihilation.

\subsection{Quantum corrections}

When the external temperature is low, and comparable to the Hawking temperature $T_{\mathrm{H}}$, the quantum correction to the effective action becomes important. The quantum part of the action, representing outside the horizon the energy of the Boulware vacuum, is given by (see for example equation (30) in Ref.23):

$$
\Omega_{\text {quantum }}=\frac{2 s+1}{192 \pi} \sqrt{-g} R \frac{1}{\partial_{\mu} g^{\mu \nu} \partial_{\nu}} R
$$

where $R$ is the Ricci scalar. If we are interested in the time independent action, then the metric $g_{\mu \nu}$ and the curvature $R$ are time independent as well. Let us first consider the space outside the horizon, where we have

$$
\partial_{\mu} g^{\mu \nu} \partial_{\nu}=\partial_{x}\left(1-v_{\mathrm{s}}^{2}\right) \partial_{x}
$$

After an integration by parts, we obtain

$$
\Omega_{\text {quantum }}(x>0)=-\frac{2 s+1}{192 \pi} \frac{1}{1-v_{\mathrm{s}}^{2}} \sqrt{-g}\left(\partial_{x}^{-1} R\right)\left(\partial_{x}^{-1} R\right)
$$

The curvature $R$ calculated by using the "acoustic" metric outside the horizon yields

$$
R=\partial_{x}^{2}\left(v_{\mathrm{s}}^{2}\right),
$$


and thus the quantum correction becomes

$$
\Omega_{\text {quantum }}(x>0)=-\frac{2 s+1}{192 \pi}\left(\partial_{x}\left(v_{\mathrm{s}}^{2}\right)\right)^{2} \frac{1}{1-v_{\mathrm{s}}^{2}} .
$$

This coincides with the result of Ref.24, obtained outside the horizon for the Boulware vacuum. Like in the case of the thermal energy, we may extend this result to the region behind the horizon. Behind the horizon, one has to consider the vacuum state as being the limit of vanishing normal component contribution (the heat bath), which keeps the value of the normal component velocity $v_{n}$ fixed. This means that we have to consider the vacuum state with respect to the moving heat bath. As a result, the coordinate dependence of the quantum action behind the horizon is determined by the counterflow velocity $w$, instead of the superfluid velocity $v_{\mathrm{s}}$. Since $w=-1 / v_{\mathrm{s}}$ (in the limit of vanishing energy flux), the result is given by (88), using the substitution $v_{\mathrm{s}}^{2} \rightarrow 1 / v_{\mathrm{s}}^{2}$ :

$$
\Omega_{\text {quantum }}(x<0)=-\frac{2 s+1}{192 \pi}\left(\partial_{x}\left(v_{\mathrm{s}}^{2}\right)\right)^{2} \frac{1}{v_{\mathrm{s}}^{2}\left(v_{\mathrm{s}}^{2}-1\right)} .
$$

Close to the horizon both regions, outside and inside the horizon, therefore contribute in a similar way:

$$
\Omega_{\text {quantum }} \approx-\frac{(2 s+1) \pi}{12} \frac{T_{\mathrm{H}}^{2}}{\left|1-v_{\mathrm{s}}^{2}\right|} .
$$

The total energy density close to the horizon, quantum + thermal, is given by

$$
\Omega(\text { total })=\Omega(\text { quantum })+\Omega(T) \approx \frac{(2 s+1) \pi}{12} \frac{T_{\infty}^{2}-T_{\mathrm{H}}^{2}}{\left|1-v_{\mathrm{s}}^{2}\right|},
$$

In agreement with more general theory (see, e.g. 24 ), the diverging terms in the thermal and quantum energy compensate each other if the external temperature at spatial infinity equals the Hawking temperature, $T_{\infty}=T_{\mathrm{H}}$, that is, in the HartleHawking state (the stress-energy tensor in the Hartle-Hawking state, defined by the vacuum of the freely falling observer, is regular at the horizon). However, despite such a compensation, the Hartle-Hawking state remains dissipative: The true temperature $T$ is inhomogeneous behind the horizon and the velocity $v_{n}$ of the normal component is nonzero. This leads to dissipation behind the horizon, due to the high frequency nonlinear dispersion.

The quantum correction does not change the entropy of the horizon in the thermal state of (80). But in the particular state of $T_{\infty}=T_{\mathrm{H}}$, the Hartle-Hawking state, the quantum correction and the entropy of the horizon are related by a general theorem. According to Refs.25.26.27, this entropy is expressed in terms of the Lagrangian as

$$
S_{\mathrm{hor}}=-8 \pi \int_{\Sigma} t_{\mu} n_{\nu} t_{\lambda} n_{\rho} \frac{\partial L}{\partial R_{\mu \nu \lambda \rho}} d \sigma
$$


In the $1+1$-dimensional case, for which $R=2 R_{0101} / g=-2 R_{0101}$, it follows that

$$
S_{\text {hor }}=-\left.8 \pi \frac{\partial \Omega}{\partial R_{0101}}\right|_{\text {hor }}=\left.16 \pi \frac{\partial \Omega}{\partial R}\right|_{\text {hor }} .
$$

Using (84) for the Lagrangian, one obtains

$$
\begin{aligned}
S_{\mathrm{hor}} & =\frac{(2 s+1)}{12}\left(\partial_{x}^{-1}\left(\frac{1}{\left|1-v_{\mathrm{s}}^{2}\right|} \partial_{x}^{-1} R\right)\right)_{\text {hor }} \\
& =-\frac{(2 s+1)}{12}\left(\ln \left|1-v_{\mathrm{s}}^{2}\right|\right)_{\text {hor }}=\frac{(2 s+1)}{18} \ln \frac{E_{\text {Planck }}}{T_{\mathrm{H}}},
\end{aligned}
$$

where we took into account the cutoff induced by the high-energy dispersion, $\mid 1-$ $v_{\mathrm{s}}^{2} \mid \sim\left(T_{\mathrm{H}} / E_{\text {Planck }}\right)^{2 / 3}$. The expression (94) coincides with the estimation of the entropy of the horizon in equation (80), if $T_{\infty}=T_{\mathrm{H}}$, i.e., for the Hartle-Hawking state.

\section{Concluding Remarks}

Horizons and ergoregions can be simulated in a multitude of different systems (cf. the overview in 28). The most promising systems, displaying quantum behaviour related to a nontrivial effective space-time, are superfluid ${ }^{4} \mathrm{He}$, superfluid ${ }^{3} \mathrm{He}$, and dilute Bose-Einstein condensates 18 229, which can be conveniently manipulated by lasers30.

The Hawking-analogous process itself is exceedingly difficult to measure in experimental practice. The Hawking temperature given by $2 \pi T_{\mathrm{H}}=\hbar \kappa_{\mathrm{s}}$ is, in experimentally relevant units, $T_{\mathrm{H}}=1.22 \mathrm{nK} \times \kappa_{\mathrm{s}}\left[10^{3} \mathrm{sec}^{-1}\right]$. In ${ }^{3} \mathrm{He}-\mathrm{A}$, where the velocity gradient attainable, in principle, is limited by $\kappa_{\mathrm{S}}<c_{\perp} / \xi_{A}$, this implies that $T_{\mathrm{H}}<0.1 \mu \mathrm{K}$. In superfluid ${ }^{4} \mathrm{He}$, the corresponding limitation, $\kappa_{\mathrm{S}}<c / \xi$, for $T_{\mathrm{H}}$ is much less severe, and comparatively large $T_{\mathrm{H}}$ would be available $\left(T_{\mathrm{H}} \sim O(10 \mathrm{mK}) \sim O\left(10^{-2} T_{c}\right)\right)$, for $\kappa_{\mathrm{s}} \sim O\left(10^{-2} c / \xi\right)$. The Hawking process would, however, probably be masked by non-Hawkingian density perturbation excitations, generated by trying to force this dense fluid to move with large gradient of velocities near the speed of sound. Matters will possibly improve for a dilute Bose-Einstein condensate, e.g. a sodium

vapour $31, T_{\mathrm{H}} \sim O(0.1 \mathrm{nK}) \sim O\left(10^{-4} T_{c}\right)$, for $\kappa_{\mathrm{s}} \sim O\left(10^{-2} c / \xi\right)$, because there to sustain this large flow gradient without generating other perturbations not related to the Hawking process might be simpler.

It appears, however, that the classical dissipative signature of a horizon for temperatures well above $T_{\mathrm{H}}$, which we have been discussing in section 5.3 , will more straightforwardly be detected with current technological means. The results of the present work can be extended to the more realistic $2+1$ and $3+1$ cases. In all cases the thin shell, which includes both sides of the horizon, is of great importance. In the shell the large blueshift is compensated by the first nonlinear correction to the linear "relativistic" energy spectrum. The thickness of the shell is much smaller than the scale of the velocity profile (the radius of the horizon in the black hole 
analogy), but also much larger than the microscopic length scale (the Planck length in the analogy). This allows for a determination of the temperature of the thermal state everywhere across the horizon. This shell is responsible for the most important properties of the thermal states in the presence of the horizon, including the entropy and entropy production, both concentrated in or close to the shell.

In principle even the 1+1-dimensional case discussed here can be realized. The condition for the applicability of our 1+1-dimensional results is that the transverse energy level separation for, say, a torus geometry of the BEC be larger than the temperature, such that these levels are not (appreciably) occupied. Such a torus trap may be realized by using a highly anisotropic trap, whose trapping frequency is "soft" in only one, ring-shaped, direction. The condition on the transverse energy level separation reads $T \ll \hbar c / 2 d$, where $d$ is the diameter of the torus of cross section $\sigma=\pi d^{2} / 4$. For that same sodium BEC we have been using for an estimation above, for $c / 2 d \sim 10^{3} \mathrm{sec}^{-1}(c \sim O(1-10 \mathrm{~mm} / \mathrm{sec}), d \sim O(\mu \mathrm{m}))$, it turns out that $T \ll 10 \mathrm{nK} \sim O\left(0.01 \cdots 0.1 T_{c}\right)$ is to be fulfilled, which appears feasible

(recently improved calorimetry has already made it possible 32 to measure temperature changes of less than $10 \mathrm{nK}$ ). A closer investigation of this classical dissipative process in these systems should, under general conditions, then take into account the effect of back-reaction on the phenomenon of horizon entropy production.

\section{Acknowledgements}

G. E. Volovik thanks Valeri Frolov and Alexei Starobinsky for helpful discussions. His work was supported by the Russian Foundation for Fundamental Research Grant No. 00-15-96699, by the Intas grant and by the European Science Foundation. U. R. Fischer was supported by the Human Capital and Mobility Programme of the European Union under Contract No. ERB FMGE CT980122 and by the DFG (FI 690/1-1).

\section{References}

1. S. W. Hawking, "Black hole explosions?", Nature 248, 30-31 (1974).

2. M. Visser, "Acoustic black holes: horizons, ergospheres, and Hawking radiation", Class. Quantum Grav. 15, 1767-1791 (1998); M. Visser, "Hawking radiation without black hole entropy", Phys. Rev. Lett. 80, 3436 (1998).

3. T. Jacobson and G. Kang, "Conformal invariance of black hole temperature", Class. Quantum Grav. 10, L201-L206 (1993).

4. W. G. Unruh, "Experimental Black-Hole Evaporation?", Phys. Rev. Lett. 46, 1351 (1981).

5. W. G. Unruh, "Sonic analogue of black holes and the effects of high frequencies on black hole evaporation", Phys. Rev. D 51, 2827-2838 (1995).

6. S. Corley and T. Jacobson, "Hawking spectrum and high frequency dispersion", Phys. Rev. D 54, 1568-1586 (1996).

7. S. Corley, "Computing the spectrum of black hole radiation in the presence of high frequency dispersion: An analytical approach", Phys. Rev. D 57, 6280-6291 (1998).

8. S. Corley and T. Jacobson, "Black hole lasers", Phys. Rev. D 59, 124011 (1999). 
9. T. Jacobson, "On the origin of the outgoing black hole modes", Phys. Rev. D 53, 7082-7088 (1996).

10. T. A. Jacobson and G. E. Volovik, "Event horizons and ergoregions in ${ }^{3} \mathrm{He} "$, Phys. Rev. D 58, 064021 (1998); T. A. Jacobson and G. E. Volovik, "Effective spacetime and Hawking radiation from a moving domain wall in a thin film of ${ }^{3} \mathrm{He}-\mathrm{A}$ ", JETP Lett. 68, 874-880 (1998) [Pis'ma Zh. Éksp. Teor. Fiz.. 68, 833-838 (1998)].

11. G.E. Volovik, "Field theory in superfluid ${ }^{3} \mathrm{He}$ : What are the lessons for particle physics, gravity and high-temperature superconductivity?", Proc. Natl. Acad. Sci. USA 96, 6042 - 6047 (1999); G.E. Volovik, "He and Universe parallelism", in "Topological Defects and the Non-Equilibrium Dynamics of Symmetry Breaking Phase Transitions", Yu. M. Bunkov, H. Godfrin (Eds.), pp. 353-387 (Kluwer, 2000).

12. I. M. Khalatnikov, "An Introduction to the Theory of Superfluidity" (Benjamin, New York, 1965).

13. A. D. Sakharov, "Vacuum Quantum Fluctuations in Curved Space and the Theory of Gravitation", Dokl. Akad. Nauk 177, 70-71 (1967) [Sov. Phys. Dokl. 12, 1040-41 (1968)].

14. G. E. Volovik, "Simulation of Painlevé-Gullstrand Black Hole in thin ${ }^{3} \mathrm{He}-\mathrm{A}$ film", JETP Lett. 69, 705-713 (1999) [Pis'ma Zh. Éksp. Teor. Fiz.. 69, 662-668 (1999)].

15. R. C. Tolman, "Relativity, Thermodynamics and Cosmology" (Clarendon Press, Oxford, 1934).

16. W. Zimdahl, "Cosmological particle production and generalized thermodynamic equilibrium", Phys. Rev. D 57, 2245-2254 (1998).

17. K. Martel and E. Poisson, "Regular coordinate systems for Schwarzschild and other spherical spacetimes", gr-qc/0001069.

18. L. J. Garay, J. R. Anglin, J. I. Cirac and P. Zoller, "Sonic analog of gravitational black holes in Bose-Einstein condensates" Phys. Rev. Lett. 85, 4643-4647 (2000).

19. M. Rice, "Superconductivity: An analogue of superfluid ${ }^{3} \mathrm{He}$ ", Nature 396, 627-629 (1998); H. Mukuda et al., "Spin-triplet superconductivity in $\mathrm{Sr}_{2} \mathrm{RuO}_{4}$ identified by ${ }^{17} \mathrm{O}$ Knight shift", Nature 396, 658-660 (1998).

20. A. F. Andreev, "The thermal conductivity of the intermediate state in superconductors", JETP 19, 1228-31 (1964) [Zh. Éksp. Th. Fiz. 46, 1823-28 (1964)].

21. G. E. Volovik, "Energy-momentum tensor of quasiparticles in the effective gravity in superfluids", gr-qc/9809081.

22. L. D. Landau and E. M. Lifshitz, "The Classical Theory of Fields" (Pergamon Press, Revised Second Edition, 1962).

23. Yu. V. Gusev and A. I. Zelnikov, "Two-dimensional effective action for matter fields coupled to the dilaton", Phys. Rev. D 61, 084010 (2000).

24. S. Mukohyama and W. Israel, "Black holes, brick walls and the Boulware state", Phys. Rev. D 58, 104005 (1998).

25. V. Iyer and R. M. Wald, "Some properties of the Noether charge and a proposal for dynamical black hole entropy", Phys. Rev. D 50, 846-864 (1994).

26. T. Jacobson, G. Kang and R. C. Myers, "On black hole entropy", Phys. Rev. D 49, 6587-6598 (1994).

27. V. Frolov and D. Fursaev, "Black Hole Entropy in Induced Gravity: Reduction to 2D Quantum Field Theory on the Horizon", Phys. Rev. D 58, 124009 (1998).

28. T. A. Jacobson, "Trans-Planckian redshifts and the substance of the space-time river", Prog. Theor. Phys. Suppl. 136, 1-17 (1999).

29. U. Leonhardt and P. Piwnicki, "Relativistic Effects of Light in Moving Media with Extremely Low Group Velocity", Phys. Rev. Lett. 84, 822-825 (2000); also cf. a Comment on this paper by M. Visser, Phys. Rev. Lett. 85, 5252 (2000), Reply ibid. 85, 5253 
(2000).

30. E. A. Cornell, J. R. Ensher and C. E. Wieman, "Experiments in Dilute Atomic Bose-Einstein Condensates", cond-mat/9903109; W. Ketterle, D. S. Durfee and D. M. Stamper-Kurn, "Making, probing and understanding Bose-Einstein condensates", cond-mat/9904034.

31. C. Raman et al., "Evidence for a Critical Velocity in a Bose-Einstein Condensed Gas", Phys. Rev. Lett. 83, 2502 (1999).

32. R. Onofrio et al., "Observation of Superfluid Flow in a Bose-Einstein Condensed Gas", Phys. Rev. Lett. 85, 2228 (2000). 